\title{
Thermal Transport in Micro and Nanoscale Systems
}

\author{
Tanmoy Maitra, Shigang Zhang and Manish K. Tiwari* \\ Nanoengineered Systems Laboratory, UCL Mechanical Engineering, University College London, Torrington Place, London WC1E 7JE, \\ United Kingdom
}

\begin{abstract}
Small scale (micro/nanoscale) heat transfer has broad and exciting range of applications. Heat transfer at small scale quite naturally is influenced - sometimes dramatically - with high surface area to volume ratios. This in effect means that heat transfer in small scale devices and systems is influenced by surface treatment and surface morphology. Importantly, interfacial dynamic effects are at least non-negligible and there is a strong potential to engineer the performance of such devices using the progress in micro and nanomanufacturing technologies. With this motivation, the emphasis here is on heat conduction and convection. The chapter starts with a broad introduction to Boltzmann Transport equation which can capture the physics of small scale heat transport and outlining the reasons why small scale transport distinct from classical micro scale heat transport. Among applications, examples are thermoelectric and thermal interface materials where micro and nanofabrication has led to impressive figure of merits and thermal management performance. Basic of phonon transport and its manipulation through nanostructuring materials are discussed in detail.

Small scale single phase convection and the crucial role it has played in developing the thermal management solutions for next generation of electronics and energy harvesting devices are discussed as next topic. Features of micro cooling platforms and physics of optimized thermal transport using micro channel manifold heat sinks are discussed in detail along with a discussion of how such systems also facilitate use of low grade, waste heat from data centres and photovoltaic modules.

Phase changes process and their control using surface micro/nanostructure are discussed next. Among the feature considered, the first are microscale heat pipes where capillary effects play an important role. Next the role of nanostructures in controlling nucleation and mobility in boiling, condensation and icing are discussed great detail. Special emphasis is placed on the limitations of current surface and device manufacture technologies while also
\end{abstract}

*Corresponding author. Tel.: +44 (0)2031081056, Email address: m.tiwari@ucl.ac.uk 
outlining the potential ways to overcome them. Lastly, the chapter is concluded with a summary and perspective on future trends and, more importantly, the opportunities for new research and applications in this exciting field. 


\section{Introduction}

Heat transfer occurs naturally between two bodies with a temperature difference. Heat transport from the hot to the cold body follows three different modes: conduction, convection and radiation. In the conduction heat transfer, heat is transported when these two objects (hot and cold) are in intimate contact with each other; heat is transported by the vibration of the object molecules. Conduction is the most significant means of heat transfer in solids. On the other hand, heat can also be transported by the movement of the fluid from one place to another, called advection. In the convection mode, heat transfer occurs by advection in combination with conduction. The movement of the fluid can be initiated naturally, for example, by the buoyancy force originating from the density difference between hot and cold part of the fluid. The fluid movement can also be forced by external means, such as a pump, a fan or a suction device. In the radiative mode of heat transport, the energy transfer occurs via an electromagnetic wave, without the need for a physical medium.

In this chapter, the discussion is limited to the heat conduction and heat convection, only. First, the basic principle of the heat transfer at a large scale are introduced with exemplifying a few practical problems where heat conduction and convection are common. Second, an extensive discussion is on heat conduction and convection at microscale regime. In this section, it has been showed that how the basic heat principle is modified to describe heat transport at small scale (micro and nanoscale regime). In the heat convection part, particularly, the discussion is split in two parts: small scale convection without and with phase change. After the subsequent discussion of each mode of heat transfer, a few applications are given where the micro/nanoscale heat transport is common. At the end, the chapter is concluded with a summary and perspective on the scope of future research.

\section{Heat conduction}

Heat conduction is commonplace in nature and manmade applications. There are plethora of industrial processes where the heat conduction is significant, such as during casting metals and their alloys (Oksman et al. 2014), polymer melt processing, heat transfer through extended surfaces ('fins'), etc. Conduction is also employed frequently in gas turbines, air conditioning units, and in cryogenic coolers where air is being used as a coolant. In these cases, heat is transported largely with the aid of convection. The low heat transfer coefficient of the air, however, limits the efficiency of these equipment and, often, extended surface area or fins (Kern and Kraus 1972 and also see chapter 12, Free Convection-External Surface) are used on the heated side to augment the heat transfer. Fourier's law is used to describe conduction heat transfer process at large scales (see also chapter 2, Macroscopic Heat Conduction 
Formulation). In the following the fundamental underpinnings of the Fourier's law, which is a phenomenological law, are discussed using the Boltzmann transport equation (BTE), which is the statistical description of the molecular motions that facilitate heat conduction.

\subsection{Introduction to Boltzmann transport equation}

The heat transfer processes such as heat conduction and convection, are described by phenomenological laws, such as the Fourier law and Newton's law of cooling in combination with the first and second law of thermodynamics (Chen 2005). These laws are typically seen as the outcome of experimental observations (Chen 2005). However, they can be derived from the statistical description of particles (molecules) facilitating the heat transfer. The distribution of particles in a material is described by a non-equilibrium function that depends on energy, temperature, and position of particles. The corresponding equation is called Boltzmann Transport equation (BTE). In the following the BTE and the derivation of Fourier's law are discussed briefly.

A particle with wave number $k$, at time $t$, is located at a position $r$. Suppose, at time $t+d t$, the particle moves to a position $r^{\prime}$ with a wave number of $k^{\prime}$ due to the existence of internal and external forces $(F)$. Then the distribution function $f(r, k, t)$ that gives the probability of finding of a particle at a particular 'state' (position and the wave number) can be solved from the following

$$
\frac{\partial f}{\partial t}+\nabla_{r} f v+\frac{1}{\hbar} F \nabla_{k} f=\left(\frac{\partial f}{\partial t}\right)_{s c a t}
$$

where $\left(\frac{\partial f}{\partial t}\right)_{\text {scat }}$ is the variation of $f$ due to scattering from the internal particles, such as electrons, photons, and phonons. Particularly, phonons, which correspond to vibration modes in a crystal lattice, play a significant role in conduction heat transfer in solids. Interestingly, it was found that phonons heat conduction at nanoscale is significantly different from their bulk counterpart, will be discussed in details later.

Particle scattering is a time dependent process, and the rigorous way to solve the scattering term $\left(\frac{\partial f}{\partial t}\right)_{\text {scat }}$ is to solve time dependent Schrodinger equations of particles. This approach makes equation 1.1 to an integro-differential equation that is extremely difficult to solve. However, a relaxation time approximation often simplifies the scattering term. This approximation assumes that if a system is thrown out of equilibrium such that $f-f_{0}$ is nonzero, collisions restore equilibrium with the dynamics following an exponential decay $f-f_{0} \approx e^{-t / \tau}$, where $f_{0}$ is the equilibrium distribution function of the particles, such as the Boltzmann, the Fermi-Dirac, and the Bose-Einstein distributions, and 
$\tau$ denotes relaxation time (the time constant for a particle to relax back to equilibrium state from a non-equilibrium state). Generally, $\tau$ is a function of $r$ and momentum ( $p$ ). The relaxation time approximation linearizes the scattering term in equation 1.1.

\subsection{Derivation of the Fourier law from BTE}

The rate of energy flow per unit area can be obtained by integrating the product of $f$, velocity vector $v(r, t)$, and the particle energy $\varepsilon(p)$ over the momentum space. With the introduction of a density of states, $D(\varepsilon)$, the energy flux $q(r, t)$ can be written as

$$
q=\int v(r, t) f(r, \varepsilon, t) \varepsilon D(\varepsilon) d \varepsilon
$$

Additionally, if $\tau$ is assumed to be independent of velocity of particles, and particle frequency, in absence of any external field (such as temperature or electric field), $f$ can be expressed as from equation 1.1

$$
f=f_{0}-\tau \frac{\partial f}{\partial t}-\tau \nabla_{r} f v
$$

Now, assuming $f$ is independent of $t$, and from equation 1.2 and 1.3, $q$ can be expressed as

$$
q=\int v \varepsilon D f_{0} d \varepsilon-\int \tau \nabla_{r} f v^{2} \varepsilon D d \varepsilon
$$

Since $f_{0}$ represents the equilibrium distribution, and it contributes an equal amount to the energy going in and out in all spatial directions, the first term of equation 1.4 drops out after the integration. Thus, equation 1.4 can be written as

$$
q=\int \tau \nabla_{r} f v^{2} \varepsilon D d \varepsilon
$$

Considering $f$ being independent of $T, \nabla_{r} f=\frac{\partial f}{\partial T}\left(\frac{d T}{d r}\right) \approx \frac{\partial f_{0}}{\partial T} \nabla T$. Now, equation 1.5 becomes,

$$
q+\tau \frac{\partial q}{\partial t}=-k \nabla T
$$


where the total thermal conductivity $(k)$ is defined as

$$
k=\int \tau v^{2} \frac{\partial f_{0}}{\partial T} D \varepsilon d \varepsilon
$$

Equation 1.6 is called Cattaneo's equation, and this derivation is based on the critical assumption of $\nabla f \approx \nabla f_{0}$, i.e., the gradient of $f$ and $f_{0}$ are the same, although $f$ changes with time. Cattaneo's equation (equation 1.6) is valid for a thermal perturbation that exists for the time scale comparable to $\tau$. However, at steady state, second term of the left side of the equation 1.6 can be neglected, and equation 1.6 simplifies to the Fourier's law of heat conduction,

$$
q=-k \nabla T
$$

\section{Heat conduction at microscale}

The progress in micro and nanotechnologies has led to a number of small-scale heat transfer devices where heat conduction plays an important role. Two broad application categories can be identified. The first application involves thermal management where the transport of heat generated within micro-/nano-scale devices such as, sensors, transducers, integrated circuits (ICs), etc. is crucial to maintain device efficiency, functionality, and reliability. Electronics cooling and semiconductors laser (laser diodes) are a few specific examples of thermal management problems. In the second type of applications, micro- and nano-structures are employed to manipulate the heat flow to maximize energy conversion efficiency; thermoelectric energy conversion and photovoltaic power generation are typical examples. In a number of these cases, Fourier's law is inadequate to describe the heat transport process, largely, because the heat transport at this scale is associated with several non-equilibrium processes occurring at a time scale much less than the $\tau$ and a length scale less than the mean free path. 


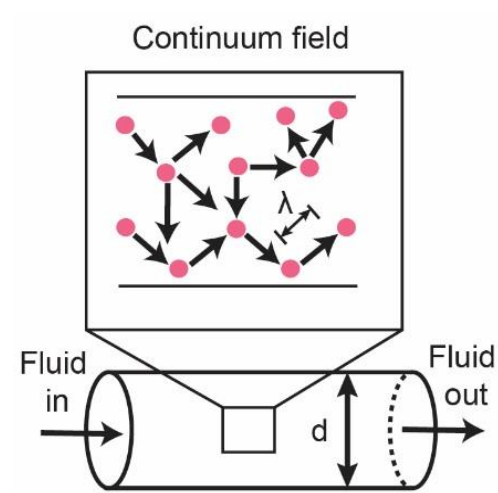

Heated pipe

Collisions are averaged out $d » \lambda$ and $t » \tau$

\section{Macroscale heat trasnfer}

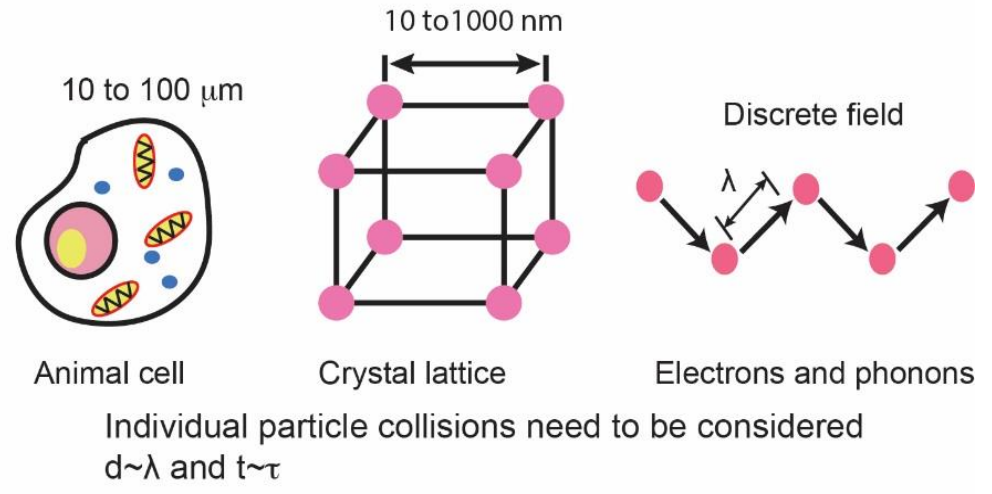

Micro/nanoscale heat trasnfer

Figure 1. Heat transfer processes occurring at different length scales: in the leftmost shows fluid flowing through a heated pip. Here, temperature field is continuous as all particle-particle interactions are averaged out over time scale $(t)$ much higher than the relaxation time $(\tau)$, towards right, temperature field is not continuous any more as the mean free path $(\lambda)$ and relaxation time $(\tau)$ approach to system dimension (d), and time scale of interest ( $t)$. Here, macroscale heat transfer analysis is not valid, and micro/nanoscale heat transfer needs to be considered.

At macroscale, all particle-particle interactions occurring at a time scale less than $\tau$ are averaged out over the time scale of interest, which is typically much higher than $\tau$ (Figure 1). Therefore, the temperature field can be assumed continuous. At microscale, however, the time scale of interest is in the same order of $\tau$, and therefore, the individual particle-particle interactions need to be considered to determine the temperature field (Figure 1). When liquid flows through a heated pipe, Fourier's law is commonly used to determine the temperature field of the liquid, and there is no need to consider the individual interactions between the fluid molecules or between the fluid molecules and the wall, since the time scale of interest is much higher than $\tau$. On the other hand, Fourier's law is inadequate to study the heat transfer mechanism, for example, in an animal cell, in a crystal lattice or electron-phonon interactions in a semiconductor (Figure 1), as the system dimension is of the order of $\lambda$. However, the general form of BTE (equation 1.1) can be utilized to study heat transport at microscale. In these cases, to describe the equilibrium distribution function $(f)$, either Fermi-Dirac or Bose-Einstein or Boltzmann distributions are used depending on type of particles under consideration. The approach enables a first principle derivation of the physical properties of materials such as thermal and electrical conductivity, heat capacity, etc. This is briefly outlined below. 
The Fermi-Dirac equilibrium distribution function is used to derive the electrical conductivity from BTE (equation 1.1). Considering the electric field, $E$ and no temperature field, the expression of electrical conductivity $(\sigma)$ can be derived from the equation of energy flux 1.2 are as follows (Chen 2005)

$$
\sigma=-\frac{2 e^{2}}{3 m_{e}} \tau\left(\mu_{F}\right) \mu_{F} D\left(\mu_{F}\right)
$$

where $\mu_{F}$ is the Fermi energy, $e$ is the electronic charge, and $m_{e}$ the mass of an electron. Here, electrons, which have energies close to Fermi energy, are contributing to the electrical conductivity of the material. Therefore, $\tau$ is calculated on the basis of only Fermi electrons.

The thermal conductivity $(k)$, however, has two parts - electron thermal conductivity $\left(k_{e}\right)$ and phonon thermal conductivity $\left(k_{L}\right)$. In metals, electrons play an important role in heat conduction, and $k_{L}$ is negligible. Considering again the Fermi-Dirac distribution function, in presence of uniform temperature gradient in one direction, and with no electric field, electron thermal conductivity can be expressed as (Chen 2005)

$$
k_{e}=\frac{2}{3 m_{e} T} \tau\left(\mu_{F}\right) \mu_{F} D\left(\mu_{F}\right) \frac{\pi^{2}\left(k_{B} T\right)^{2}}{3}
$$

where $k_{B}$ is the Boltzmann constant, and $T$ is the temperature field along one direction. Like the derivation of $\sigma$, electrons that have energies close to Fermi energy, only contribute to the electronic thermal conductivity.

Unlike metals, electrons play insignificant role in heat conduction in semiconductors and insulators, and therefore the total thermal conductivity, $k$ is approximated to the phonon thermal conductivity, $k_{L}$, which can be calculated by the following equation.

Considering Kinetic theory, and the Bose-Einstein distributions, $f_{B E}$ (as phonons are Bosons), $k_{L}$ can be expressed as (Chen 2005)

$$
k_{L}=\frac{1}{3} \int_{0}^{v_{m}} \tau v_{a}^{2} h v \frac{\partial f_{B E}}{\partial T} D(v) d v
$$

where $v_{m}$ is the maximum Debye frequency and defines the maximum frequency of the phonon in the crystal, $v_{a}$ is the average velocity of a phonon. The detail derivation of equations 1.10 and 1.11 can be found elsewhere (Chen 2005). 
Assuming $\tau$ and $v_{a}$ are independent of temperature, the remaining part in the integral of equation 1.11 is the specific heat per unit volume, which can be expressed as

$$
c_{v}=\int_{0}^{\infty} \hbar v \frac{\partial f_{B E}}{\partial T} D(v) d v
$$

Studies show that $c_{v}$ has a complex dependency on $T$. At low temperature, $c_{v}$ depends on $T^{3}$. At high temperature, however, $c_{v}$ depends on $T^{-n}$, where $n=1-1.5$.

The physical properties captured by equations 1.8-1.11, thermal conductivity in particular, are amenable to change by suitably engineering the materials composition and/or dimension, which directly affect the density of states. This offers some unique and exciting potential applications in the field of energy and nanotechnology. In next two sections, it will be shown how such micro- and nano-structures are employed to manipulate heat conduction at small scales in two exemplar applications, namely thermoelectric and thermal interface materials.

\subsection{Thermoelectricity}

The above microscale heat transport analysis is of direct relevance to thermoelectricity where inter-conversion of electrical and thermal energy is achieved without using any moving parts. The direct interconversion between heat and electricity can be uniquely valuable in a number of applications such as exploitation of waste/low-grade heat to generate electricity, refrigeration and cooling without moving parts etc. Although, the principle has been known since long (DiSalvo 1999), limitation of the thermoelectric materials to rare earth materials was a major shortcoming, not to mention the low energy efficiency of these devices. However, with development of micro- and nano-fabrication techniques significant advances were achieved very recently - in the last few decades (Poudel et al. 2008; Vineis et al. 2010). The idea of micro/nanostructuring started with pioneering calculations and modeling in the early 90 's showed that due to quantum confinement of electrons in low dimensional - micro-/nanostructured - materials, $s^{2} \sigma$ can be increased independently (Hicks and Dresselhaus 1993; Hicks et al. 1993). Interestingly, when the dimension of materials is comparable to phonon's mean free path (50-300 nm at room temperature) (Balandin and Nika 2012), the phonon transport is dominated by the boundary scattering. Importantly, in this case, phonon thermal conductivity directly scales as the system dimension. Moreover, in low dimensional materials, electron mobility is also adversely affected by the spatial confinement of phonons. Thus, nanostructuring and, consequently, by altering phonon's boundary scattering can be used to engineer phonon thermal conductivity and electrical conductivity of any material 
(Ziman 2001). Given the fact that silicon is the second most abundant material on the earth and, thanks to progress in microelectronics industry, has established micro/nano-fabrication protocols, it has been widely explored as a thermoelectric material.

The performance of a thermoelectric materials is quantified by a unitless figure of merit $(Z T)$, which is defined as

$\frac{\sigma S^{2} T}{k}$, where $s$ is the Seebeck coefficient. In most of material systems, $\sigma, s$ and $k$ are interrelated, and therefore, each parameter cannot be changed without altering others. This interdependence and counterbalancing trends make it difficult to enhance $Z T$ and therefore, this are an active area of research. Advances made to this end with micro/nanostructuring are discussed in the following subsections, which capture the broad strategies employed for improving the ZT coefficient.

\subsubsection{Low dimensional materials: superlattices and nanowires}

Superlattices are 2D (layered) nanostructured materials (see Figure 2), which were the first to be used as a low dimensional thermoelectric material. Numerous superlattice material systems such as $\mathrm{Bi}_{2} \mathrm{Te}_{3} / \mathrm{Sb}_{2} \mathrm{Te}_{3}$ (Venkatasubramanian et al. 2001), $\mathrm{PbTe} / \mathrm{PbSe}_{\mathrm{x}} \mathrm{Te}_{1-\mathrm{x}}$ (Vineis et al. 2010), $\mathrm{Si} / \mathrm{Si}_{1-\mathrm{x}-\mathrm{y}} \mathrm{Ge}_{\mathrm{x}} \mathrm{C}_{\mathrm{y}}$ (Fan et al. 2001; Vashaee and Shakouri 2007) etc. have been established to increase $Z T$. Some researchers have also shown that $Z T$ can be further augmented by decreasing superlattice periods down to $3 \mathrm{~nm}$ (Harman et al. 1999). In this context, Luckyanova et al. were first to establish that quantum confinement in superlattices significantly affect the phonon transport (Luckyanova et al. 2012) and thus the conduction process. In fact, the phonon transport in superlattices can be either coherent or incoherent. For example, phonons traverse internal region of the film of superlattices ballistically leading to a coherent transport, whereas incoherent phonon transport occurs at the boundary of superlattices as phonons are scattered diffusely at the boundary. In most superlattices, incoherent phonon transport dominates over the coherent transport. The phase information of phonons is lost in the incoherent transport. However, the periodicity of superlattices can control the phonon transport, which in turn influences phonon's thermal conductivity; thus, raising the possibility of engineering the thermoelectric performances (Casimir 1938; Cahill et al. 2003). In fact, Luckynova et al. (Luckyanova et al. 2012) showed that at a temperature below $150 \mathrm{~K}$, the thermal conductivity of superlattices increases as the number of lattice periods increase, as shown in Figure 2A and B). Figure 2C shows that thermal conductivities 
increases as the number of lattice periods increases up to the temperature of $150 \mathrm{~K}$ indicating the dominance of ballistic transport of phonons.
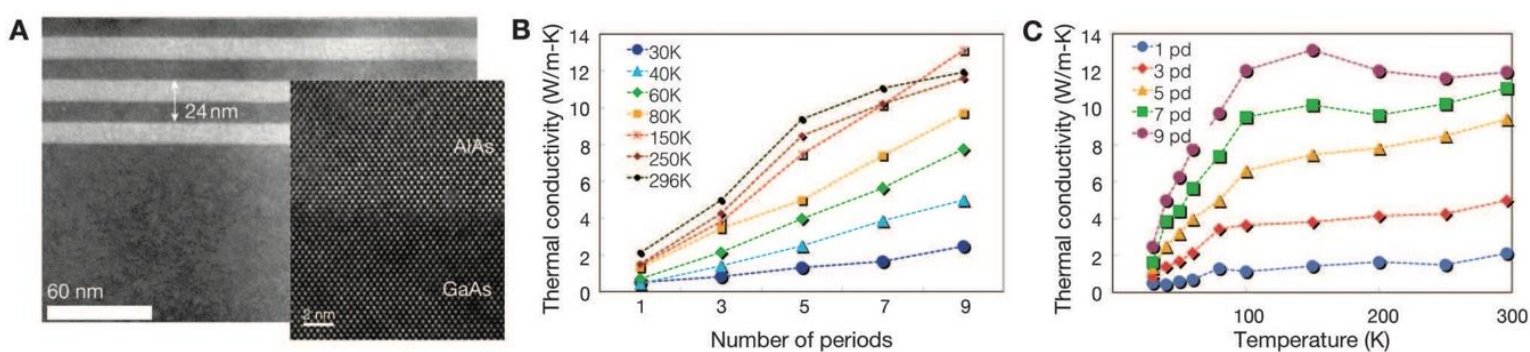

Figure 2. Thermal conductivities of superlattices: (A) Cross-sectional Transmission Electron Microscopy (TEM) image of AlAs/GaA 3-period superlattice. Inset shows the high resolution TEM image at the interface of GaAs and AIAs. (B) Variation of experimentally measured thermal conductivity at different temperatures of superlattices with varying periods. (C) Variation of thermal conductivity with temperature at different number of lattice periods (Reproduced by the permission of The American Association of the Advanced of Science (Luckyanova et al. 2012)).

Boundary scattering also extends to one dimension (1D, nanowires of a material). Lower thermal conductivities of nanowires compared to the bulk are achieved because the transport of electrons and phonons, particularly those, which have mean free path in the same order of magnitude as that of nanowires diameters, is severely impeded. However, phonons that have smaller mean free path compared to diameters of nanowires are practically unaffected. Therefore, there is a possibility to reduce further thermal conductivities of nanowires by suppressing the scattering of those short length phonons. One possible approach to deplete those phonons is to reduce nanowires diameters. There has been active research into thinner nanowires for thermoelectrics, as for example group III and IV nanowires, to further enhance the quantum confinement of electrons (Li et al. 2003; Lin and Dresselhaus 2003). In this regard, experimental and theoretical study indicated that indeed higher $Z T$ values can be obtained with thinner nanowires, although fabricating very thin nanowires, down to $5 \mathrm{~nm}$, is a fabrication challenge. To this end, the prevalence of silicon $(\mathrm{Si})$ in microelectronics and the related micro/nanomanufacturing knowhow, has raised exciting possibilities of using them as efficient thermoelectric materials. Thus, the thermal and electrical properties of Si nanowires have been studied widely. Figure 3 shows an example of work by demonstrating the thermal conductivity reduction in Si nanowire. Although subsequently improved through roughening the nanowires (Figure 3A and B, see also the next subsection), Li et al. (Li et al. 2003) were the first to synthesize 'smooth' Si nanowires using vapor-liquid-solid technique and show that with reduction in wire diameter the thermal conductivity could be reduced by two orders of magnitude compared to bulk (black symbols in Figure 3C). 

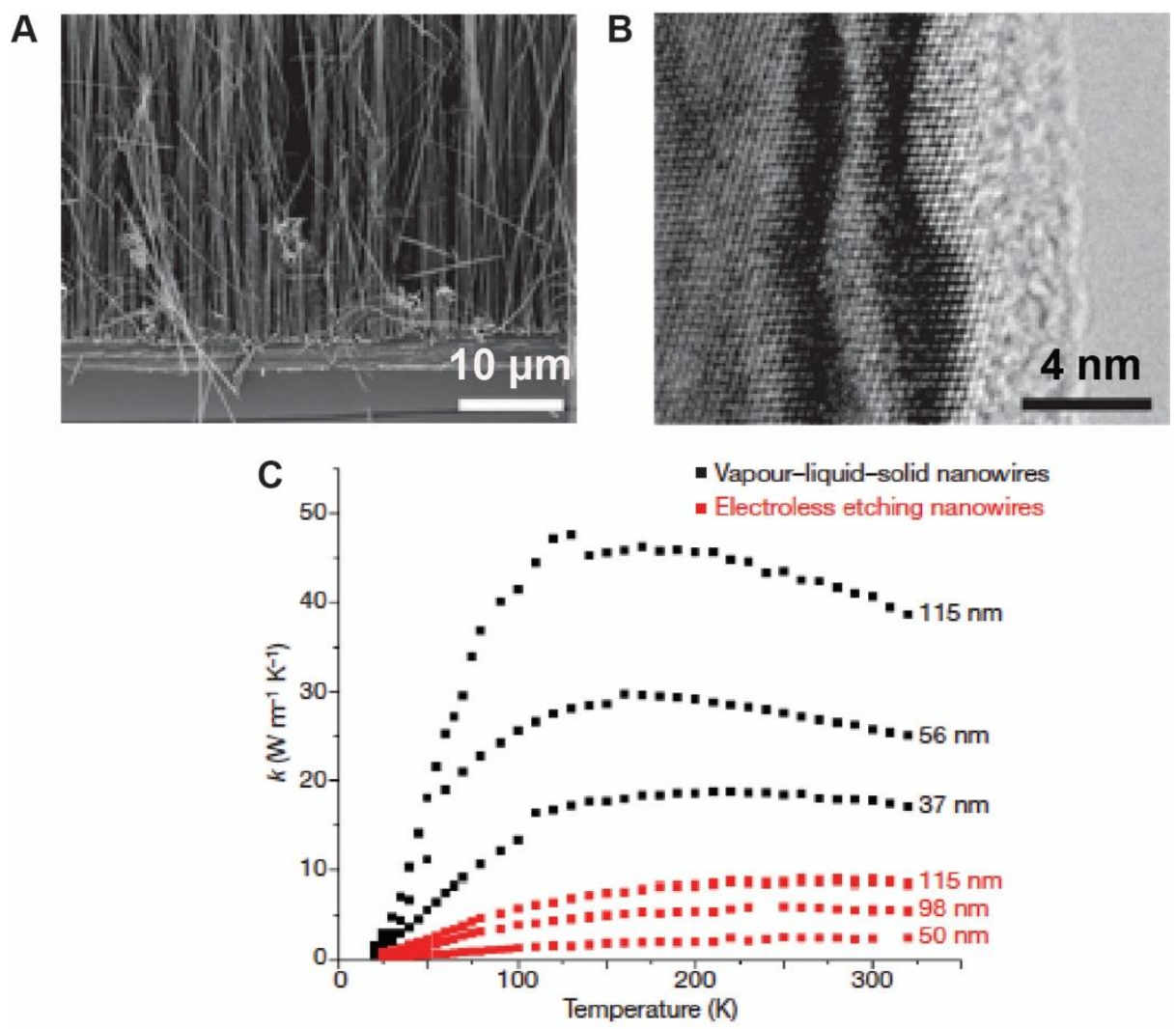

Figure 3. Silicon nanowires for thermoelectrics: (A) Cross-sectional SEM image of silicon nanowires, (B) High resolution Transmission Electron microscopic (TEM) image of silicon nanowires after the surface treatment. The roughness of silicon nanowires is clearly visible in the TEM image. (C) Experimentally measured thermal conductivities of such roughened silicon nanowires of different diameters as a function of temperatures. Silicon nanowires are produced two different techniques, vaporliquid-solid (VLS), and electroless etching (EE) techniques. Reproduced by the permission of Nature Publishing group (Hochbaum et al. 2008) and American Institute of Physics (Li et al. 2003).

\subsubsection{Nanostructuring: at surface and bulk}

Nanostructuring is an additional strategy which can introduce numerous grain boundaries and interfaces in the materials, which enhance phonon boundary scattering and, thus, lead to a significantly reduction in thermal conductivity and enhancement of ZT (Rowe et al. 1981; Dresselhaus et al. 2007; Lan et al. 2010). Therefore, nanostructuring has been applied not only to low dimensional materials (e.g. nanowires) but also to bulk nanograined materials (Lan et al. 2010). Holchbaum et al. 2008) showed that surface nanostructuring of silicon nanowires led to a significant reduction in thermal conductivities due to enhanced phonon boundary scattering. These rough $\mathrm{Si}$ nanowires were produced by electroless etching technique, and roughened through surface treatment, which is evident from the high resolution TEM image in Figure 3B. Figure 3C shows the effect of diameters of silicon nanowires on experimentally measured thermal conductivities at different temperatures. Below $150 \mathrm{~K}$ and at a particular nanowire 
diameter, the thermal conductivities of roughened silicon nanowires are smaller compared to those nanowires that are not surface treated, and produced by vapor-liquid-solid (VLS) technique (Li et al. 2003). The roughness/surface nanostructure was investigated further by Lim et al. (Lim et al. 2012). They fabricated silicon nanowires by VLS method, and subsequently employed two different etching processes - one comprising galvanic deposition of Silver at the surface boundaries of nanowires followed by etching with hydrofluoric acid (HF), and the other focusing on wet etching in the mixture of $\mathrm{HF} / \mathrm{H}_{2} \mathrm{O}_{2}$ and $\mathrm{AgNO}_{3}$ - induced two distinctly unique surface roughnesses. The study presented a quantitative relationship between the nanowire length, diameters and root mean square roughness, and thermal conductivities.
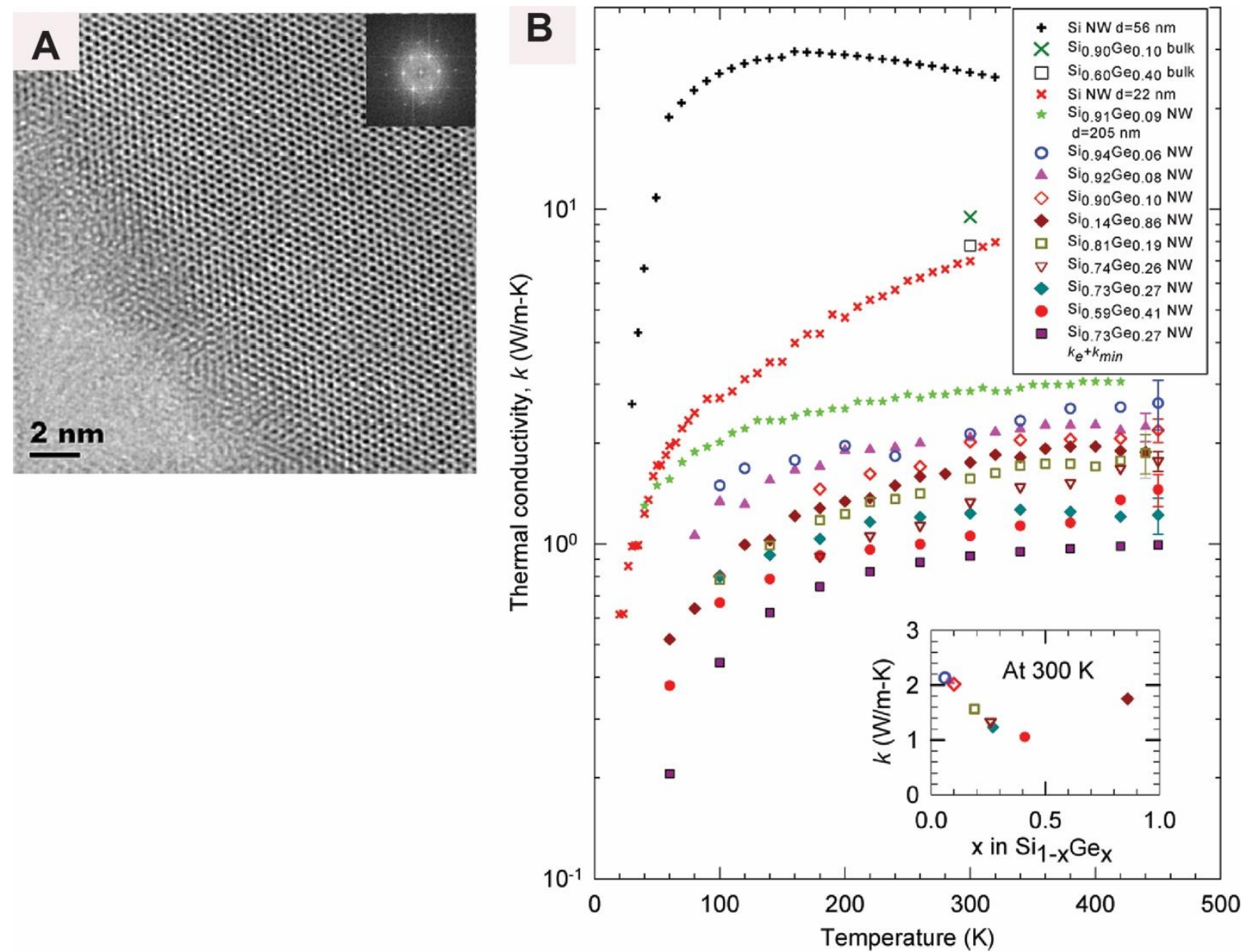

Figure 4. Silicon-Germanium (Si-Ge) composite nanowires: (A) High resolution TEM image of Si-Ge composite nanowire. Inset shows electron diffraction pattern obtained from a selected area of Si-Ge nanowire. (B) Thermal conductivities of bulk Si-Ge nanowires at varying Ge content in Si at different temperatures, reproduced from Dismukes et al. 1964; Li et al. 2003; Kim et al. 2010b. Inset shows the thermal conductivities of Si-Ge bulk nanowires at different Ge content as performed by Lee et al. (Lee et al. 2012). Reproduced by the permission of American Chemical Society ((Lee et al. 2012)). 
Another strategy to reduce thermal conductivity is to introduce defects, in the form of impurities, to affect those short length phonons (Lee et al. 2012; Perez-Taborda et al. 2016). Lee et al.(Lee et al. 2012) showed a significant reduction of thermal conductivities of silicon nanowires by alloying with Germanium (Ge). Figure 4A a high resolution TEM image Si-Ge composite nanowire, clearly showing the grain boundary between the two components. Figure 4B plots thermal conductivities of $\mathrm{Si}-\mathrm{Ge}$ composite nanowires (with different compositions), synthesized from bulk composites. It is clear from experimental data that the effect of Ge content on the overall thermal conductivities are apparent below $100 \mathrm{~K}$ temperature. However, $\sigma$ and $S$ are also sensitive to impurities. Therefore, Lee et al. also measured thermal conductivities as well as $\sigma$ and $S$ simultaneously from the same nanowires. Impressively, bulk SiGe composite nanowires showed a significantly improved $Z T$, around 0.46 at $450 \mathrm{~K}$.

The advances using 2D and 1D nanostructured materials clearly demonstrate a path to engineer thermoelectric materials. However, scalability to large systems is an issue. Bulk nanostructured materials are an alternative and address the issue of scalability. The approach was elegantly demonstrated by Poudel et al.(Poudel et al. 2008), who introduced bulk alloy BiSbTe as efficient thermoelectric materials. Essentially, nanopowders were prepared by simple ball milling of a block of p-type BiSbTe, followed by hot pressing them to form a bulk ingot with a large number of scattering grain boundaries. The thermal conductivity of the resulting nanostructured ingot was significantly lower than its homogenous counterpart and, impressively, a $Z T$ value of 1.4 at $100^{\circ} \mathrm{C}$ was achieved. The approach is by no means limited to BiSbTe; systematic reviews document research on materials, establishing the generality of this approach (Minnich et al. 2009; Sootsman et al. 2009).

In addition to extensive use of inorganic materials, there have also been a number of reports focusing on alternate materials such as conjugated organic polymers as thermoelectric materials. An immediate advantage of using organic polymers are light weight, good mechanical properties, and low production cost (McGrail et al. 2015), which are desirable in realizing the next generation of large scale flexible electronic devices and also to realize light weight power sources. Conjugated organic polymers such as, polyaniline (Kaneko et al. 1993), polyacetylene (Yoon et al. 1995; Mateeva et al. 1998), polypyrrole (Kemp et al. 1999), polythiphenes (Hu et al. 2013) etc., show a good electrical conductivity by charge delocalization across the polymer backbone. To enhance thermoelectric performances (ZT), electrical conductivity of organic polymer needs further improvement. This can be achieved by blending with other 
polymers (Kim et al. 2013), nanoparticles (Moriarty et al. 2013; Kim et al. 2010), inorganic salts (Sun et al. 2011; Bissessur et al. 1993) etc.

\subsubsection{Doping}
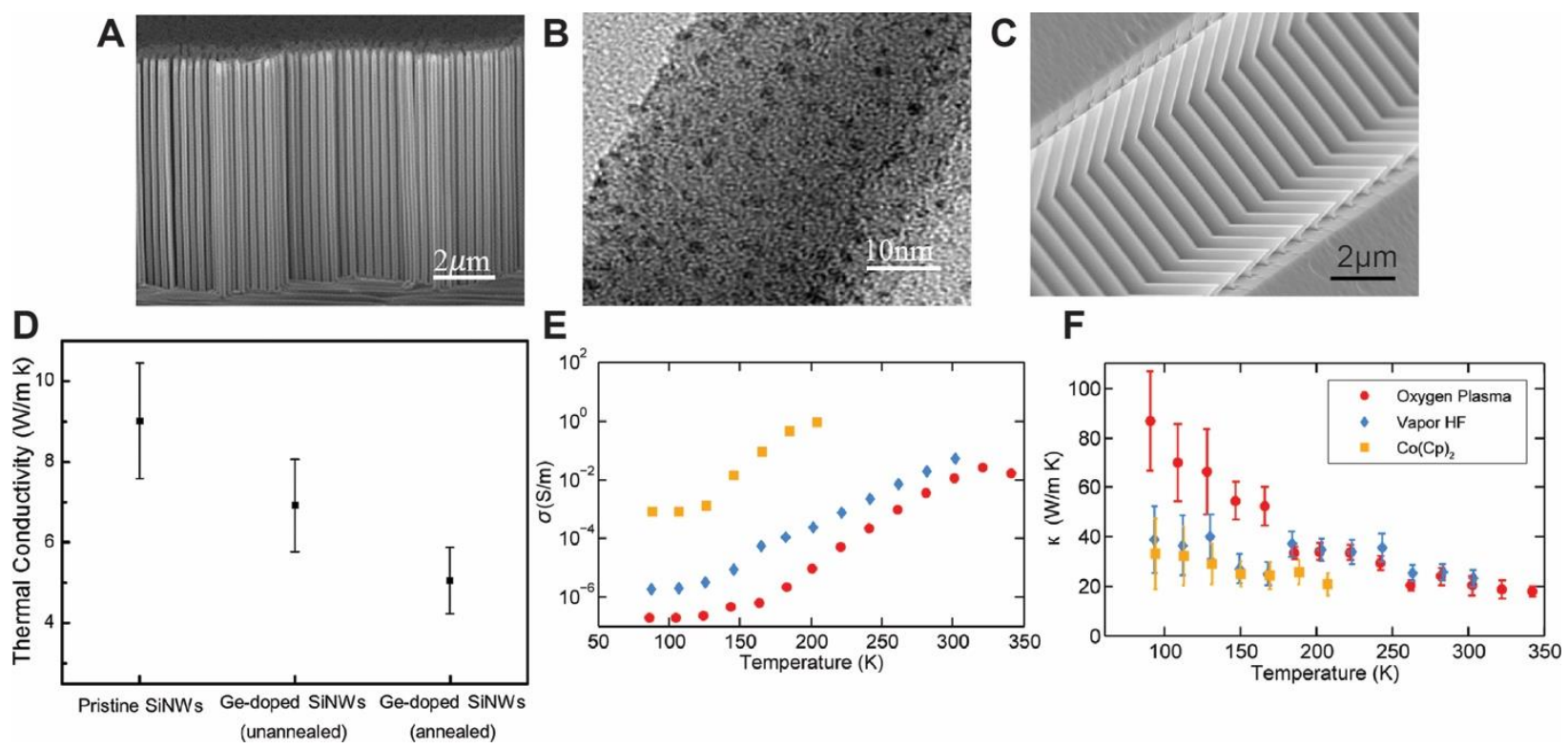

Figure 5. Doped silicon nanowires: (A) Scanning Electron Microscopic (SEM image of the Silicon nanowire forest, (B) High Resolution TEM image of a Germanium-doped Silicon nanowire, (C) SEM image of free standing Silicon nanowires, (D) Thermal conductivity measurements of pristine silicon nanowire and Ge-doped silicon nanowires. (E) and (F) electrical and thermal conductivity measurements of free standing silicon nanowires with different surface treatments, such as oxygen plasma, vapor $\mathrm{HF}$ and doping with $\mathrm{Co}(\mathrm{Cp})_{2}$. Reproduced by the permission of American Institute of Physics(Pan et al. 2015) and American Chemical Society(Pan et al. 2016).

Given the ease of nanofabrication with $\mathrm{Si}$, numerous attempts have been made to modify $\mathrm{Si}$ nanowires, either by doping with other p-type and n-type materials or altering the surface chemistry, to augment the electrical conductivity, and simultaneously to reduce the thermal conductivity (Donadio and Galli 2009; Huang et al. 2007; Balasubramanian et al. 2011; Pokatilov et al. 2005; Pan et al. 2016; Pan et al. 2015). In this regard, Pan et al. (Pan et al. 2015) fabricated a silicon nanowire forest via a simple technique, using polystyrene spheres as sacrificial templates and followed by a gold-assisted silicon etching technique. The fabricated silicon nanowires are coated with Germanium (Ge), a n-type dopant, and followed by a thermal annealing step to enhance the interaction between Ge and Si. An SEM image of such a silicon nanowire forest and a high resolution TEM image of Ge-doped silicon nanowires are shown in Figure 5A and B. Experimental measurements, as shown in Figure 5D, clearly indicate a reduction in thermal conductivities 
of silicon nanowires after doping with Ge, and a further reduction after the thermal annealing step. Thermal conductivity and electrical conductivity measurements have also been performed by the same research group (Pan et al. 2016) on a free standing silicon nanowires where nanowires were suspended between a heating and sensing pad. A SEM image of such free standing silicon nanowires are presented in Figure 5C. Interestingly, surfaces of free standing nanowires are modified by different surface modification techniques, such as oxygen plasma, vapor phase HF treatment and n-type surface charge transfer doping. The experimental results, Figure 5E and F, showed an enhancement of electrical conductivity, and a simultaneous reduction in thermal conductivity of surface modified silicon nanowires. In addition, the n-type surface charge transfer doped silicon nanowires had a higher electrical conductivity and a lower thermal conductivity at a specific temperature compared the nanowire modified by the remaining techniques (Pan et al. 2016).

Generally, the reduction in thermal conductivities due to the surface doping results from the enhancement of the incoherent phonon transport. The effect can also be observed with thin silicon films. For example, Asheghi et al. (2002) reported $80 \%$ reduction in thermal conductivity of doped silicon compared to pure crystalline silicon. Dopants, however, also increase the number of electrons (with n-type dopants) and holes (p-type dopants), resulting in a potential decrease in $\sigma$ and $s$, and, thus, ZT. Note the dopants also increase $k_{e}$, relatively modest influence on the overall thermal conductivity (Vineis et al. 2010). Therefore, use of dopants requires a careful trade-off (Vineis et al. 2010).

\subsection{Thermal interface materials}

A thermal interface material (TIM) facilitates heat conduction between two components. TIMs have plethora of industrial applications where generated waste heat needs to be transferred effectively to heat sinks. In these applications, TIMs provide a conductive path from one materials (heat source) to the other (heat sinks). Therefore, thermal resistance and mechanical property, particularly stiffness, are two crucial metrics of materials to be used as a TIM. The thermal resistance of TIMs needs to be low (Marconnet et al. 2013). Stiffness of TIMs, on the other hand, needs to be low to accommodate the mismatch of thermal expansion between hot and cold components (Marconnet et al. 2013). Generally, metals have low thermal resistances, but high stiffness. Polymers, on the contrary, possess low stiffness, but high thermal resistances. Industries have been using different materials like solders (metal alloys), thermal pastes (polymer composites with high thermal conductance), and phase change materials. However, the high thermal resistances offered by those commercially available materials merely satisfy the current industry's need, 
particularly for microelectronic industry where the need for miniaturization and high computing speeds lead to the production of huge amount of process heat within a very small area. Nanostructured materials could be a promising future candidate for TIMs due to their extraordinary physical properties, particularly high thermal conductivity and superior mechanical properties: the most exciting candidates are discussed in more detail next.
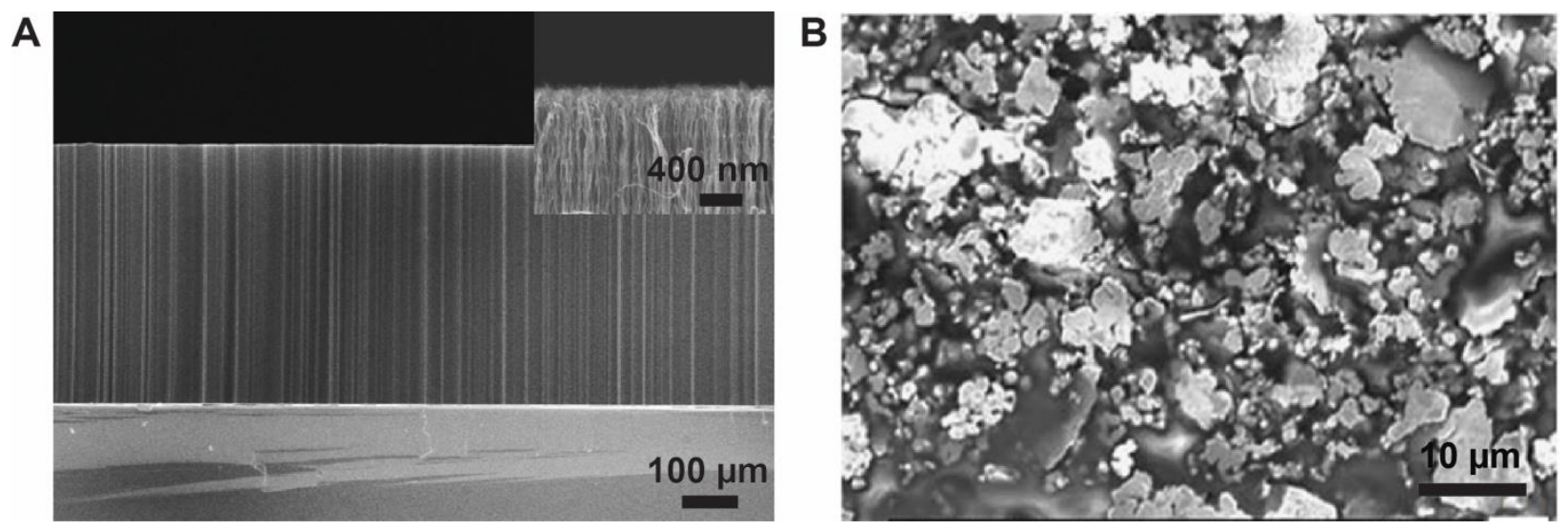

Figure 6: Carbon nanotube (CNT) and graphene based polymer composite thermal interface materials: (A) Vertically aligned CNT forest used as a thermal interface material (TIM). Inset shows the high resolution of SEM image of CNT forest, some CNTS are bended at the interface resulting to poorer contacts, and high boundary scatterings. (B) SEM image of graphene-based TIM. Reproduced by the permission of Elsevier Masson SAS (McNamara et al. 2012b; Shahil and Balandin 2011)

\subsubsection{Carbon nanotube and other nanoparticles in polymer matrix}

The common nanostructured TIMs are fabricated by combining nanoparticles fillers with superior thermal conductivities and polymers with lower stiffness. To this end, carbon nanotubes (CNT), i.e. $\mathrm{sp}^{2}$ hybridized carbon allotrope, which are formed by rollup of single or multi-layer graphene (planar layer of hexagonally arranged carbon atoms), has elicited great interest as filler particles due to its very high conductivity. The experimental investigations on the evaluation of thermal performances of CNT/polymer composite, however, only show moderate increase in the effective thermal conductivity compared to the theoretically predicted values. The discrepancy can be attributed (McNamara et al. 2012a) to:

1. random dispersion of CNTs in the polymer matrix; i.e. not all CNTs are participating in the heat conduction event

2. inadequate CNT loading and/or poor dispersion quality of CNT in the polymer

3. high boundary resistance between CNT and polymer 
The effective thermal conductivity of the polymer-CNT composite can be enhanced by improving the dispersion quality (Choi et al. 2003; Marconnet et al. 2011).

Theoretical studies indicate that vertically aligned CNTs (VACNT) (Balandin 2011; McNamara et al. 2012b; Marconnet et al. 2013) standalone, as shown in Figure 6A, may be used as TIM due to higher intrinsic conductivity of CNTs along their axial direction. CNTs were grown directly on metal substrates, and thermal measurements indeed showed higher effective thermal conductivity compared to polymer-CNT composite. However, there are some issues with VACNT that limits their practical exploitation (McNamara et al. 2012a):

1. Mean free path of phonons in CNT is $1.5 \mu \mathrm{m}$, and the area of contact between CNT and substrate only a few $\mathrm{nm}$. Therefore, the ballistic transport of phonon is important along the axial direction of the CNT. However, the measured thermal conductance is much smaller than the actual thermal conductance of CNT. This discrepancy is largely due to the higher CNT-boundary resistance. Phonons scatter at the boundary (see section 2.1), and thus increase the boundary resistance.

2. CNT are mostly grown by chemical vapor deposition (CVD) technique. The resulting CNTs are not exactly the same length, and therefore some CNT cannot form a good mechanical contact to substrates due to bending at the interface (inset of Figure 6A).

3. Inherent acoustic mismatch between CNT and substrates lead to a high phonon scattering.

4. Adhesion between substrates and CNT are weak resulting in a weak heat conduction path.

Potential strategies to overcome the issue of poor CNT/substrate contact include chemical modification of substrates (Lin et al. 2008; Lin et al. 2010); which also helps limit phonon's boundary scattering.

\subsubsection{Graphene based TIMs}

Graphene, one atom thick sp ${ }^{2}$ hybridized carbon atoms arranged in a planner sheet, has the higher thermal conductivity than CNT (Shahil and Balandin 2012). Therefore, graphene, as an alternative to CNT, can be used as TIM. In fact, graphene based polymer composites (Error! Reference source not found.B) show better thermal conductivity compared to CNT-polymer composites. This superior behavior is due to the availability of $\pi$ electrons on graphene surfaces (since graphene is $\mathrm{sp}^{2}$ hybridized) and the planner geometry graphene. The latter facilitates better contacts between graphene and polymer matrix, and thus phonon scattering at the boundary of graphene decreases (Shahil and Balandin 2012). However, the effective thermal conductivity of graphene-polymer composite depends on various 
parameters, such as surface roughness of graphene, its quality (single or double layer etc.) etc.(Minnich et al. 2009), which need to be controlled carefully (Shahil and Balandin 2012; Shahil and Balandin 2011).

\section{Heat convection}

In the previous section, the discussion was centered on the heat conduction where the molecular distribution and vibration are important. However, the heat can also be transported through the movement of fluid, i.e. by convection. For example, a hot liquid is flowing through a pipe, heat up the pipe wall. Generally, Reynolds number $(R e)-$ ratio of the inertial force to the viscous force - is used to characterize the fluid flow. In small scales, focused herein (for discussion on large scale convection, see chapter 8. Single-Phase Convective Heat Transfer), the viscous forces dominate and $R e$ is small; examples include polymer extrusion processing, electronic cooling, etc. In extrusion processing (Pearson 1985), a polymer is melted and pushed through a metallic channel and an orifice of a given shape. The viscous dissipation is high due to the low flow rate, and the high viscosity of the polymer melt. Interestingly, heat convection at low $R e$ is also important in the Geophysics, where the flow of the earth's mantle is studied. The mantle (Birctt 1952) is the viscous earth's interior. The temperature of the mantle can be as high as $500{ }^{\circ} \mathrm{C}$. The heat is transported from the earth's interior to the surface by convection. As the mantle is viscous and it flows slowly, the convective heat transfer resistance is higher than the conductive one (Gebhart 2017; Birctt 1952). Under conditions of low $R e$ the governing physics and equations are amenable to some simplifications, which are discussed in greater details next.

\subsection{Governing equations and dimensionless numbers in heat convection}

For the incompressible flow, in a steady state process, continuity equation can be written (Beek and Muttzall 1975)

$$
\nabla v=0
$$

With constant density, the fluid momentum is governed Navier-Stokes equation, which equates rate of change of fluid momentum with net forces on a fluid element and can be written as (Beek and Muttzall 1975)

$$
\rho \frac{D v}{D t}=-\nabla p-\nabla \tau
$$

where $\frac{D}{D t}$ is called substantial time derivative, and is defined as (Beek and Muttzall 1975) 


$$
\frac{D}{D t}=\frac{\partial}{\partial t}+\Sigma v_{i} \frac{\partial}{\partial i}
$$

with $v_{i}$ denoting the velocity of the fluid in $i^{\text {th }}$ direction. First term of the left hand side in equation 1.14 represents the inertial force; the first and the second term in the right hand side denote pressure and viscous force, respectively. At steady state, and for Newtonian fluid, equation 1. 14 yields (Beek and Muttzall 1975)

$$
\rho(v . \nabla v)=-\nabla p+\nabla(\mu . \nabla v)
$$

where $\mu$ denotes the fluid viscosity.

Neglecting potential energy and kinetic energy, the thermal energy balance equation can be written (Beek and Muttzall 1975)

$$
\rho \frac{D U}{D t}=-(\nabla \cdot q)-\nabla(p \cdot v)-\nabla \cdot\left(\tau^{\prime} \cdot v\right)
$$

$U, q$ and $\tau^{\prime}$ denote internal energy, amount of heat energy input by conduction, and shear stress of the fluid, respectively. Considering $U=U(V, T)$, at constant pressure and incompressible liquid, the left hand side of equation 1. 17 becomes,

$$
\rho \frac{D U}{D t}=\rho c_{p} \frac{D T}{D t}
$$

Combining equations 1.17 and 1.18 yields

$$
\rho c_{p} \frac{D T}{D t}=\mathrm{k} \nabla^{2} T-\nabla(\tau . v)
$$

The last term on the left hand side of equation 1. 19 is the energy term associated with viscous dissipation, and can be represented via a function of velocity gradients (Beek and Muttzall 1975), $\mu \Phi_{v}$, where $\Phi_{v}$ denotes the so called dissipation function. Therefore, equation 1. 19 becomes,

$$
\rho c_{p} \frac{D T}{D t}=\mathrm{k} \nabla^{2} T-\mu \Phi_{v}
$$


Let us define dimensionless terms $T, v, t$ and $\check{x}$ as

$T^{*}=\frac{T}{\Delta T} ; v^{*}=\frac{v}{\check{v}} ; t^{*}=\frac{t}{(D / \bar{v})}$ and $\check{x}=\frac{x}{D}$

where $\Delta T, \check{v}$ and $D$ denote the characteristic temperature differential, velocity and length scale, respectively. With the introduction of these non-dimensional terms, equation 1. 20 becomes (Yazicioglu and Kakaç 2010),

$$
\frac{D T^{*}}{D t^{*}}=\frac{1}{P e} \nabla^{* 2} T^{*}+\frac{B r}{P e} \Phi^{*}
$$

where Peclet number $(P e)$, the product of Reynolds number $(R e)$ and Prandtl number $(P r)$, signifies the relative importance of convective heat transfer in the fluid and heat conduction from the wall to the fluid. At low $R e, P e$ can be made higher by reducing the characteristics system dimension $(D)$. Brinkman number $(B r)$ is the ratio of viscous dissipation to heat conduction from the wall to the fluid. At low $R e, B r$ becomes relevant as the axial conduction becomes important. The sections following next will focus on application specific use and simplification of the above equations.

\subsection{Single phase convection at microscale}

Microscale heat convection has many applications such as cooling of micro/nanoelectronics, photovoltaic cells, and laser diodes, etc. Newton's law, $q=h \Delta T$ where $h$ denotes the heat transfer coefficient of a fluid, describes overall performance of heat convection processes (Kothadran 2011). The heat transfer coefficient $h$ is obtained by solving the Navier-Stokes (momentum) and energy equations, with an assumption of no fluid slippage at solid walls. However, at microscale, the boundary conditions may need modification, depending on the Knudsen number (Kn) (Yazicioglu and Kakaç 2010), which is defined as the ratio of mean free path of fluid molecules $\lambda$ to the characteristic length scale of the system, e.g., the channel diameter $D$. Essentially, the $K n$ is used to characterize the flow regime. If $K n<0.001$, flow can be considered to be continuum where conventional no-slip condition at the wall is maintained. For $0.001<$ $K n<10$, e.g., in rarefied flows, were are in a 'transition' regime, and no-slip condition is not valid anymore. When $K n>10$, the flow is dominated by molecular diffusion and the heat flux and temperature gradient cannot maintain a linear relationship. In fact, the fluid and the solid cannot retain thermodynamic equilibrium at the interface and the no-slip condition is not valid. Navier-Stokes equations, however, can be applied with the 'Slip' boundary condition. 
As an additional feature, as the system dimension becomes smaller, $P e$ also gets smaller leading to a higher convective heat transfer resistance inside the fluid as compared to the conductive heat transfer resistance. Furthermore, at the small scale, the viscous dissipation becomes significant, and hence $B r$ becomes large. In general, at small scale, flow can be assumed to be laminar (as $R e$ is low), and for fully developed flow, $h$ can be obtained from the correlation (Panigrahi 2015) (see chapter 9, Forced Convection- Internal Flow),

$$
N u=\frac{h D}{k}=3.657
$$

From equation 1. 23, it is clear that $h$ scales inversely as $D$. Therefore, $h$ is high at small (micro/nano) scales. The decrease in convective heat transfer resistance, however, is at the expense of the higher pressure drop, which increases the fluid pumping power and, therefore, affects the overall efficiency of the process. One particular application of microscale convection and a topic of major scientific in last two decades is electronic cooling. Salient aspects of this application will be discussed next.

\subsubsection{Electronics cooling}

The heat generated in microelectronic systems needs to be dissipated effectively and often under severely constrained geometries, due in large part to the steady progress in device miniaturization. This need has led to a broad and active research into thermal management using micro/nanoscale cooling platforms, and led to a new field of research termed 'electronics cooling' (Koo et al. 2005). Electronics cooling covers theoretical and experimental investigations on thermal management - which is naturally not just limited to microelectronics industry - and assessment and characterization of thermal reliability of the cooling hardware. Efficient thermal management offers immediate advantages in efficiency, robustness, and power handling capability of microelectronics. There are a number of different approaches to convective electronic cooling.

An effective cooling strategies is jet impinging cooling (Kandlikar and Bapat 2017) where high-speed jets issues from nozzles and the microchips are kept at a certain distance away from and perpendicular to the nozzles. A thin boundary layer is formed immediately under the jet, and this boundary layer becomes thicker as the liquid starts to flow radially outward direction. Therefore, due to the variation of the boundary layer thicknesses, the convection coefficient $h$ also varies from the center to the edge of the microchip and, thereby, producing large temperature gradients in the 
microchip. Due to this, thermal stresses in the chip to the heat sink interface increases, and electronic reliability, particularly in hotter regions of the chip, reduces. This is a notable drawback of jet impinging cooling.

Spray cooling (Kandlikar and Bapat 2017) is another strategy. This process is based on impinging followed by evaporation of the liquid droplets. As this cooling strategy relies on the liquid to vapor phase change process, a large amount of heat can be removed at much lower surface temperatures. This is an immediate advantage. Spray cooling efficiency can be further enhanced by atomizing the fluid into smaller drops, for example, by using pressure-assisted atomizing nozzles. The high pressure requirement in such nozzles is, however, poses a mechanical reliability challenge. A third strategy relies on using fluid flow through microchannels for cooling. Microchannel cooling (Tuckerman and Pease 1981; Escher et al. 2010b; Sharma et al. 2013) offers high heat transfer coefficient due to the smaller channel width, as discussed above. Common coolants used are air and water (Khonsue 2012; Wang and ChiChuan 2017). However, water has three orders of magnitude higher density, four times higher specific heat and an order of magnitude better thermal conductivity compared to air. This facilitates remarkable improvement in heat transfer efficacy and enables designs of compact heat sinks for cooling microprocessors, for example (Alfieri et al. 2010; Escher et al. 2010a). In fact, microchannel based single phase cooling have been exploited to remove a large amount of heat, as high as $750 \mathrm{~W} / \mathrm{cm}^{2}$ (Sharma et al. 2013). Unfortunately, the associated higher pressure drop limits the use of microchannel cooling. To overcome this issue and to tackle the challenge of cooling 3D chips stacks (see section 3.2.1.2 below), two important cooling platforms have been introduced, as outlined below.

\subsubsection{Microchannel geometry: Manifold microchannel (MMC)}

To reduce the pressure drop of the liquid across the microchannel, a manifold microchannel design is adopted where liquid, instead of being delivered at a particular inlet, is delivered across the chip through uniformly spaced nozzles. The nozzles are each part of a manifold sitting on top of the microchannels. Thus the manifold layers distribute the flow and the flow from the nozzle outlet impinges on to the microchannels underneath. The arrangement is referred as manifold microchannel (MMC) heat sink, as shown in Figure 7A, and reduces the travel length of the liquid through microchannel, and hence, significantly decreases the pressure drop. A number of studies (Escher et al. 2010a; Escher et al. 2010b; Sharma et al. 2013; Sharma et al. 2015a; Sharma et al. 2015b) have also been done to optimize manifold and channel geometries in MMC heat sinks to enhance the overall cooling efficiency by reducing the pressure drop. One example of such an optimized cooling strategy is the hotspot targeted microchannel cooling. This strategy is 
based on the delivery of cooling liquid to a high-heat flux region, and throttling the liquid flow in a low heat flux region. With this strategy, much more uniform chip temperature can be attained while consuming low pumping power, and thus enhance the efficiency of the overall cooling process.

The effectiveness of MMC heat sinks and significantly better thermal properties of water can be exploited to facilitate reuse the waste heat from energy intensive components such as data centers, concentrated photovoltaic systems, etc. This helps to remarkably improve the overall thermal performance and energy efficiency of these systems. In fact, a recent study showed that the heat recovery efficiency can be as high as $80 \%$ using MMC heat sinks with water as a coolant (Kasten et al. 2010; Zimmermann et al. 2012a; Zimmermann et al. 2012b; Tiwari et al. 2012). Interestingly, these works used coolant water temperature of $\sim 60^{\circ} \mathrm{C}$, i.e. well above the ambient temperature, to cool microprocessor chips. Zimmermann et al. (Zimmermann et al. 2012b) performed detailed exergy and feasibility analysis of such a hot water MMC heat sinks. They showed that raising the coolant inlet temperature was an effective way to ensure that even hotter water coming out of the data center was useful in secondary usage such as district heat, adsorption cooling, membrane based water desalination, etc. The hot water based cooling strategy opens up a new avenue as it enables us to use efficiently otherwise wasted heat in datacenters and offers not only an improved thermal performance of a datacenters as whole also a possible elimination of chiller unit used in datacenters to precool air in warm climates.

The MMC heat sinks have also been used to cool concentrated photovoltaic (CPV) cells (Zimmermann et al. 2015). Photovoltaic chip efficiency goes down with increase in chip temperature, thus cooling is beneficial. The problem is exacerbated in concentrated photovoltaic chips, where solar light is focused down to a small area to save the expensive chip material by exploiting optical components which are cheaper. For CPVs operating at a high concentration, a high heat flux is produced. Consequently, an efficient heat sink is needed for cooling. Just as in the case of data centre cooling mentioned above, Zimmermann et al. 2015 showed that using efficient MMC heat sinks CPVs could be cooled even with hot water. The high inlet cooling temperature only marginally affected the electrical efficiency of the photovoltaic solar cell module. However, crucially, it produced even hotter water at the outlet of the heat sink which could be used in secondary applications such as building heating, thereby boosting the system energy efficiency. Impressively, the strategy enabled a fourfold increase in the overall energy efficiency, from $15 \%$ to $60 \%$ (Zimmermann et al. 2015). 


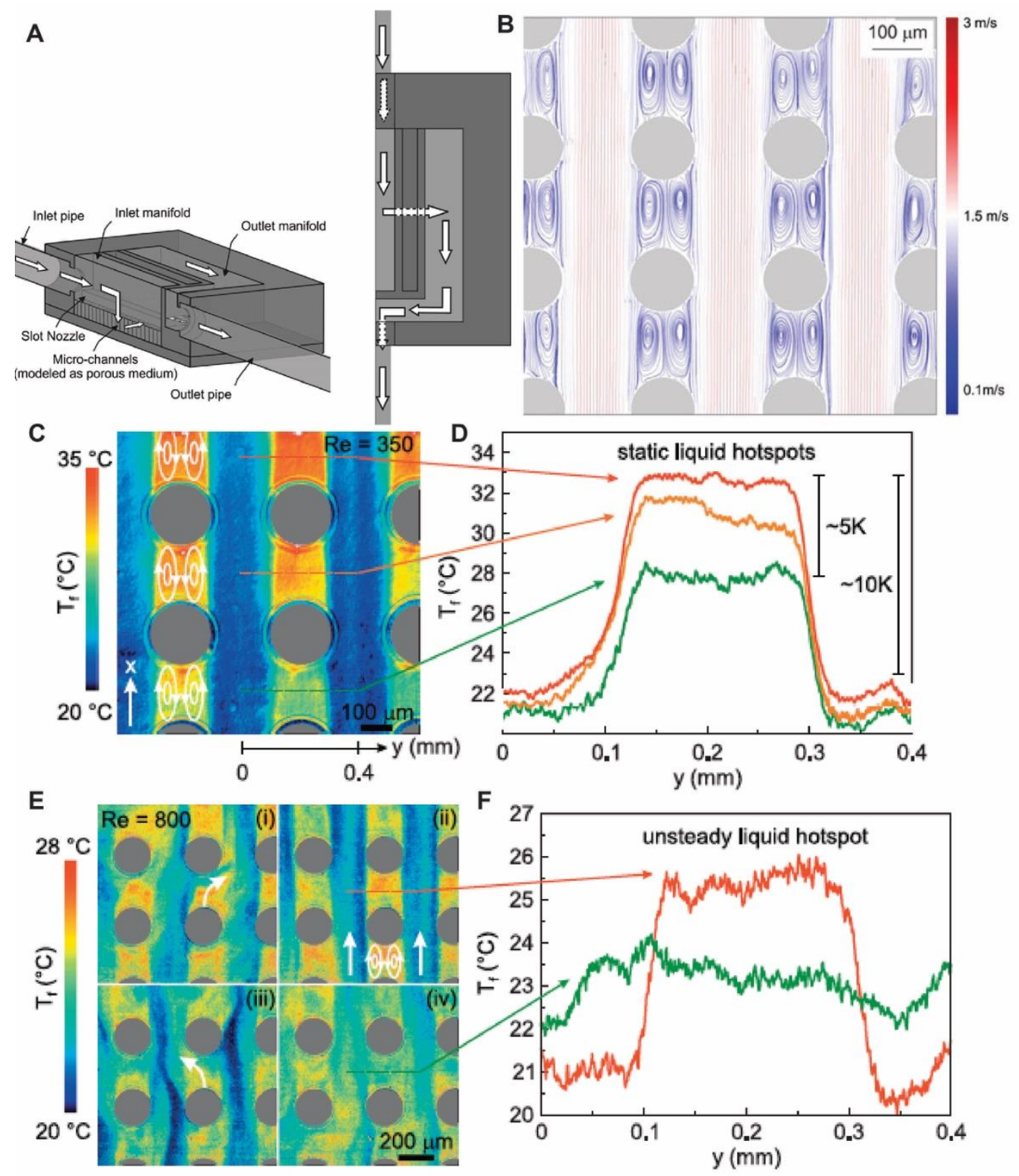

Figure 7. Flow and heat transfer in water cooled heat sinks: (A) Schematic of manifold microchannel (MMC) heat sink in isometric view and top view. (B) Streamline pattern of the steady flow across a chip with inline micropin array. At Re 170, a stable pair of vortices is formed between the pins in the flow direction. (C) Liquid temperature map on a micropin fin based heat sink in the prevortex flow regime. (D) Transversal temperature profile at three different locations, as indicated by three different arrows. (E) Image sequences of instantaneous temperature map in the post-vortex regime showing gradual destruction of liquid recirculation zones. (F) Transversal temperature profile at two different locations, as indicated by two arrows. Reproduced by the permission of Elsevier Ltd. (Renfer et al. 2013; Sharma et al. 2013) and Springer Verlag (Renfer et al. 2011). 


\subsubsection{Microchannel geometry: 3D architecture}

Recently, electronics industry is adapting 3D integrated circuit (IC) design to accommodate more functionalities into a small space, lower the overall cost by partitioning large chip into multiple smaller dies, and reduce IC wire length. In 3D ICs, microprocessor cores are stacked on top of each other, with through silicon vias (holes) in each core that are filled with conductive materials to connect the cores together. The strategy greatly reduces the data transfer time between cores. However, this compact 3D design brings with it additional challenges. Higher junction temperature, and accessibility of cooling fluids to each layer are two common challenges in 3D IC design. Therefore, a smarter and stronger cooling strategy is needed to address these challenges. Researchers have proposed interlayer integrated chip cooling as a possible strategy (Renfer et al. 2011). In such integrated cooling approach, cooling hardware (e.g. microchannels, micropins, etc.) are embedded at the back of each chip layer. Typically, this comprises of a microcavity with micropins (representing the vias). This arrangement is adopted to seal the electrical interconnects from water. However, the hydrodynamics of the flow of the cooling liquid may be influenced by the embedding micropin array in microcavity. Renfer et al. indeed (Renfer et al. 2011) showed that with increase in flow Re there was a clear sign of steady (Figure 7B) and shedding vortices in the wake of cylinder. This flow transition was found to be associated with a fluctuation in the flow field, and a sharp transition in the pressure drop trend. The authors also performed visualization of the fluid flow field and its relevant effects on the temperature and pressure distribution of the fluid in the micropin array heat sinks (Renfer et al. 2013). They reported instantaneous temperature maps of the micropin heat sink in pre-vortex and post-vortex shedding stages using the microscale Laser Induced Fluorescence $(\mu \mathrm{LIF})$ technique (Renfer et al. 2013), which is a popular technique to study the role of microscale flow on convective heat transfer. The temperature map of a micropin based heat sink obtained by the $\mu$ LIF is shown in Figure 7C to F. Interestingly, in the pre-vortex transition regime, at $R e \sim 350$ (calculated based the mean velocity between a pair of pins) a stagnant recirculation zone was formed behind micropins, and a stable microchannel like flow occurs in between the pins (Figure 7C). The stagnant recirculation zones had a higher temperature than the zones where microchannel like flow occurred. Figure 7D shows transversal temperature profile at three different locations, extracted from the $\mu$ LIF maps. The temperature of recirculation zones increases rapidly by $5 \mathrm{~K}$ over three pins in the direction of the flow whereas the temperature only increases by $1 \mathrm{~K}$ in the free liquid stream (see Figure 7D). In addition, the temperature difference between recirculation and microchannel type flow zones is $10 \mathrm{~K}$. In the post-vortex regime, at $R e \sim 800$, the shedding vortices dynamically alter the temperature maps, as shown in image sequences in Figure 7E (i) to (iv), and leading to 
advection of liquid hotspots into the main flow stream. Note also that the laminar boundary layer, which is formed in the post transition regime, is destroyed due to the vortex-induced liquid mixing. The complete destruction of liquid recirculation zones and the laminar boundary layer result a uniform liquid temperature field (Figure 7F), and nearly a two-fold enhancement in heat transfer performances (Renfer et al. 2013).

\subsection{Two phase convection at microscale}

In phase change processes, the major amount of heat is transported in the form of the latent heat. Typically, the latent heat is much larger than the sensible heat (i.e. heat transferred due to temperature difference). Therefore, the heat transfer is drastically enhanced compared to the single phase approach. Due to this superior performance, phase change processes are desired in many energy applications, such as thermal generation of electricity, desalination, different metallurgical processes, electronic cooling, food processing, etc. Although the phase change processes are used widely in industry, the coexistence of two phases and their effects on the thermal transport are not fully understood, despite a few good propositions (Qu and Mudawar 2003; Lee and Mudawar 2005). However, empirical studies have enabled substantial improvement in the performance of two-phase processes, thanks in major part to the advancement of micro/nano-fabrication techniques. Heat pipe is simplest of heat transfer devices relying on phase change, recent advances have led to micro heat pipes for passive thermal management. Additional phase change processes are also employed; among these boiling, condensation and icing have benefitted significantly from developments in novel fabrication technologies. Some relevance phase change devices and their underlying physics are discussed next.

\subsubsection{Micro heat pipe}
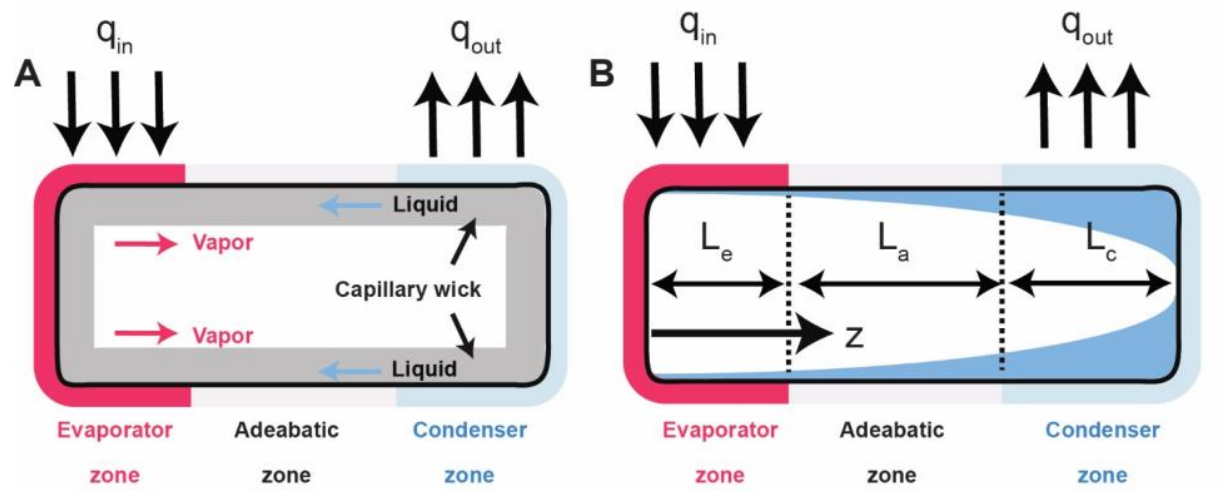

C

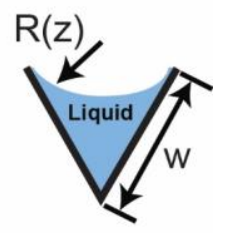

Figure 8. Different types of heat pipes: Schematic of $(A)$ a conventional heat pipe with a capillary wick, (B) a capillary wickless micro heat pipe, and (c) liquid meniscus at a corner in the micro heat pipe. 
A heat pipe is a heat transfer device and used frequently for thermal management in space craft, computers, solar collector array, air conditioning system etc. It relies on two phase heat transfer and comprises an evaporation zone, an adiabatic zone, a condensation zone, a liquid (coolant), and a wick structure, as shown in Figure 8A (see also chapter 54, Heat Pipes and Thermosyphons). The heat pipe is generally made of high thermal conductivity material, such as copper, aluminum etc. The internal wall of a standard heat pipe is covered with a porous (wick) structure to facilitate the liquid wicking, and to provide the surface area for the heat transfer. The center of the heat pipe remains hollow to facilitate the flow of the evaporating fluid from the evaporation to the condensation zone through the adiabatic zone. The working liquid is chosen depending on the operational temperature of the pipe. After the removal of the air, the heat pipe is partially filled with the saturated coolant liquid and sealed. When the heat is absorbed in one end of the heat pipe, the liquid in the wick of the evaporation zone evaporates. The vapor fills up the center, diffuses along the length of the pipe, and finally reaches the condensation zone. The temperature of the condensation zone is kept slightly below the saturation temperature of the liquid, and therefore, vapor condenses with the release of the latent heat. The transport of the liquid from the condensation to the evaporation zone relies on the surface texture and wettability of the wick, and occurs passively without an active control. This is the major advantage of heat pipes and enables compact system designs. Many researchers have investigated on design of wick structures to facilitate constant supply of the coolant to the heated surface, and routing the vapor phase evolved during the phase change process. Examples include, a porous wick structure, e.g., sintered copper (Weibel et al. 2010), different microstructures (Chen et al. 2016), etc., with good wettability towards coolant liquid. Such wick structures, however, provide no control of the evolved vapor, which can saturate the porous materials, particularly at high heat flux, and block further liquid transport. It is even more complicated to control the liquid transport at a small scale (micro- and nano-scale) compared to the relatively large scale (submicron to millimeter). Micro- and nanoporous wick structure provides more surface area per unit volume to the heat transfer. However, this can also lead to a large amount of vapor production in the porous wick structure and block the coolant flow, causing an early evaporation dry out may occur (Semenic et al. 2008; Rice and Faghri 2007; Weibel et al. 2010). Despite these challenges, heat pipes find plethora of applications in small scale devices in electronics cooling, passive thermal management of electrical devices, solar thermal energy technology to name but a few. Small scale heat pipes are referred as micro heat pipe (MHP). MHPs are capillarity (interfacial force) driven heat pipes where the mean curvature of vapor/liquid interface is comparable to the reciprocal 
of hydraulic radius of the channel and were first introduced by Cotter in 1984 (Cotter 1984a). In contrast to a conventional heat pipe, an MHP has no capillary wick. Rather, the capillary action of the coolant relies on the sharpangle corners in a polygonal microchannel. The coolant condenses at the corners in the condensation zone of the MHP, as depicted in Figure 8B. The liquid meniscus curvature is different between the evaporation and condensation zones, thereby helping to create a pressure differential (due to Laplace pressure) which can drive the liquid coolant. The maximum heat transfer capacity is reached when simultaneously dry out and flooding transpire in the evaporation and condenser zone, respectively. The thermal performance of an MHP is often evaluated on the basis of transient energy and mass balance, which are discussed below.

Heat transfer in solid wall of the MHP

Energy balance equations on the wall of MHP can be written as

$$
\rho_{s} c_{p, s} \frac{\partial T}{\partial t}=k_{s} \nabla^{2} T+S
$$

where $\rho_{s}, c_{p, s}$ and $k_{s}$ denote the density, heat capacity, and thermal conductivity of the MHP wall, respectively. The symbol $S$ denotes the heat gained/lost by the wall at any z-coordinate, and for different MHP sections (see Figure 8B), can be expressed as (Cotter 1984a; Carbajal et al. 2006; Liu and Chen 2013)

$$
\begin{array}{ccr}
S=-h_{l}\left(T_{s}-T_{l}\right) w_{b}+q_{\text {in }} l_{o}, & 0 \leq z \leq L_{e} & 1.24 \\
S=-h_{l}\left(T_{s}-T_{l}\right) w_{b}, & L_{e} \leq z \leq L_{e}+L_{a} & 1.25 \\
S=-h_{l}\left(T_{S}-T_{l}\right) w_{b}-j h_{f g}+h_{o}\left(T_{s}-T_{c}\right), & L_{e}+L_{a} \leq z \leq L_{e}+L_{a}+L_{c} & 1.26
\end{array}
$$

where $h_{l}$ and $h_{o}$ are convective heat transfer coefficient of liquid, respectively, to the evaporation zone and the outer wall of the condenser; $T_{s}, T_{l}$ and $T_{c}$ denote the temperatures of the solid wall, liquid and the cooling water used at the condensing outer wall to extract heat, respectively; $q_{\text {in }}$ and $q_{\text {out }}$ are the heat input in the evaporation and condenser zone, respectively; $w_{b}$ is the wetted perimeter of the MHP grooves; and $j$ and $h_{f g}$ are the condensing mass flux and latent heat of evaporation, respectively. The condensation flux $j$ can be expressed as (Liu and Chen 2013; Carbajal et al. 2006)

$$
j=\left(\frac{2 \widehat{\sigma}}{2-\widehat{\sigma}}\right)\left(\frac{M}{2 \pi R_{u}}\right)^{0.5}\left(\frac{p_{v}}{T_{v}{ }^{0.5}}-\frac{p_{l}}{T_{l}^{0.5}}\right)
$$


where $\hat{\sigma}, M, R_{u}, p_{v}$ and $p_{l}$, respectively, denote accommodation factor, molecular weight of the vapor, and universal gas constant, the vapor pressure and saturate vapor pressure of the liquid at the operating temperature of the MHP.

\section{Capillary flow in the evaporation zone}

Capillary radius at the liquid/vapor interface can be calculated from Young-Laplace equation, as follows

$$
\Delta p=\gamma_{l-v}\left(\frac{1}{R_{1}}+\frac{1}{R_{2}}\right)
$$

where $\Delta p, \gamma_{l-v}, R_{1}$ and $R_{2}$, respectively, denote pressure differential across the liquid/vapor interface, liquid/vapor interfacial energy, and radii of curvature in the axial and radial directions. $R_{2}$ is much larger than $R_{1}$ and, therefore, equation 1.28 can be approximated as,

$$
\Delta p \approx \gamma_{l-v}\left(\frac{1}{R_{1}}\right)
$$

For a constant vapor pressure of the liquid inside the MHP, equation 1. 29 can be written in differential variation of $p_{l}$ as

$$
\frac{d\left(p_{l}\right)}{d z}=\frac{\gamma_{l-v}}{R_{1}^{2}} \frac{d R}{d z}
$$

This pressure gradient is capillarity induced and helps drives the liquid flow in MHP (Liu and Chen 2013). The mass balance equations for the liquid flow in the MHP can be written as (Cotter 1984a; Carbajal et al. 2006; Liu and Chen 2013)

$$
\begin{gathered}
\frac{\partial\left(\rho_{l} A_{l}\right)}{\partial t}=\frac{\partial\left(\rho_{l} A_{l} V_{l}\right)}{\partial z}-M_{l}, 0 \leq z \leq L_{e} \\
\frac{\partial\left(\rho_{l} A_{l}\right)}{\partial t}=\frac{\partial\left(\rho_{l} A_{l} V_{l}\right)}{\partial z}, L_{e} \leq z \leq L_{e}+L_{a} \\
\frac{\partial\left(\rho_{l} A_{l}\right)}{\partial t}=\frac{\partial\left(\rho_{l} A_{l} V_{l}\right)}{\partial z}-M_{v}, L_{e}+L_{a} \leq z \leq L_{e}+L_{a}+L_{c}
\end{gathered}
$$


where $A_{l}$ is the cross-sectional area for liquid flow, $V_{l}$ the liquid velocity, $\rho_{l}$ its density, $M_{l}$ and $M_{v}$ are the masses of the liquid evaporated and condensed per unit length and time. Likewise, transient energy balance in evaporator, adiabatic and condenser zone can be written as follows (Cotter 1984b; Carbajal et al. 2006; Liu and Chen 2013)

$$
\rho_{l} c_{p, l} A_{l} \frac{\partial T_{l}}{\partial t}=A_{l} k_{l} \nabla^{2} T_{l}+\rho_{l} c_{p, l} A_{l} \nabla T_{l}+h_{l}\left(T_{s}-T_{l}\right) w_{b}-M_{l} j
$$

where $k_{l}$ and $c_{p, l}$ denote the thermal conductivity and specific heat of the coolant liquid.

The above mass and energy balance equations are used to evaluate the performance of MHPs. In addition to the capillary forces, the external force like electric field can also be employed to enhance the liquid motion inside the MHP. In those techniques, free space charges are introduced in the liquid coolant either by direct injecting a small amount of mobile charges through an emitter tip (ion injection pump) (Chang and Yeo 2010; Chang and Hung 2017) or by the dissociation of ions upon the application of the electric field (conduction pump) (Chang and Yeo 2010; Chang and Hung 2017). Those ions act as free charges, initiate the electrohydrodynamic flow in the MHP in presence of the electric field. Alternatively, an electrode is introduced along the axial direction of the MHP to generate polarization forces driving the dielectric coolant liquid into a non-uniform electric field. Therefore, a hydrostatic equilibrium is established in the coolant liquid (induction pump) (Jones 1973). Typically, the thermal conductance of induction pump based MHP is lower than MHPs based on the ion injection or conduction pump mechanisms (Chang and Hung 2017).

\subsubsection{Microscale boiling}

Boiling occurs when the temperature of a liquid is higher than its saturation temperature. Considering a classic case of a liquid in contact with a solid, depending on the solid-liquid temperature difference (i.e. the degree of superheat), the liquid boiling progresses through different regimes as will be described in brief below. The boiling regimes are depicted in Figure 9A where heat flux $q$ is plotted as a function of degree of superheat $\Delta T$. 


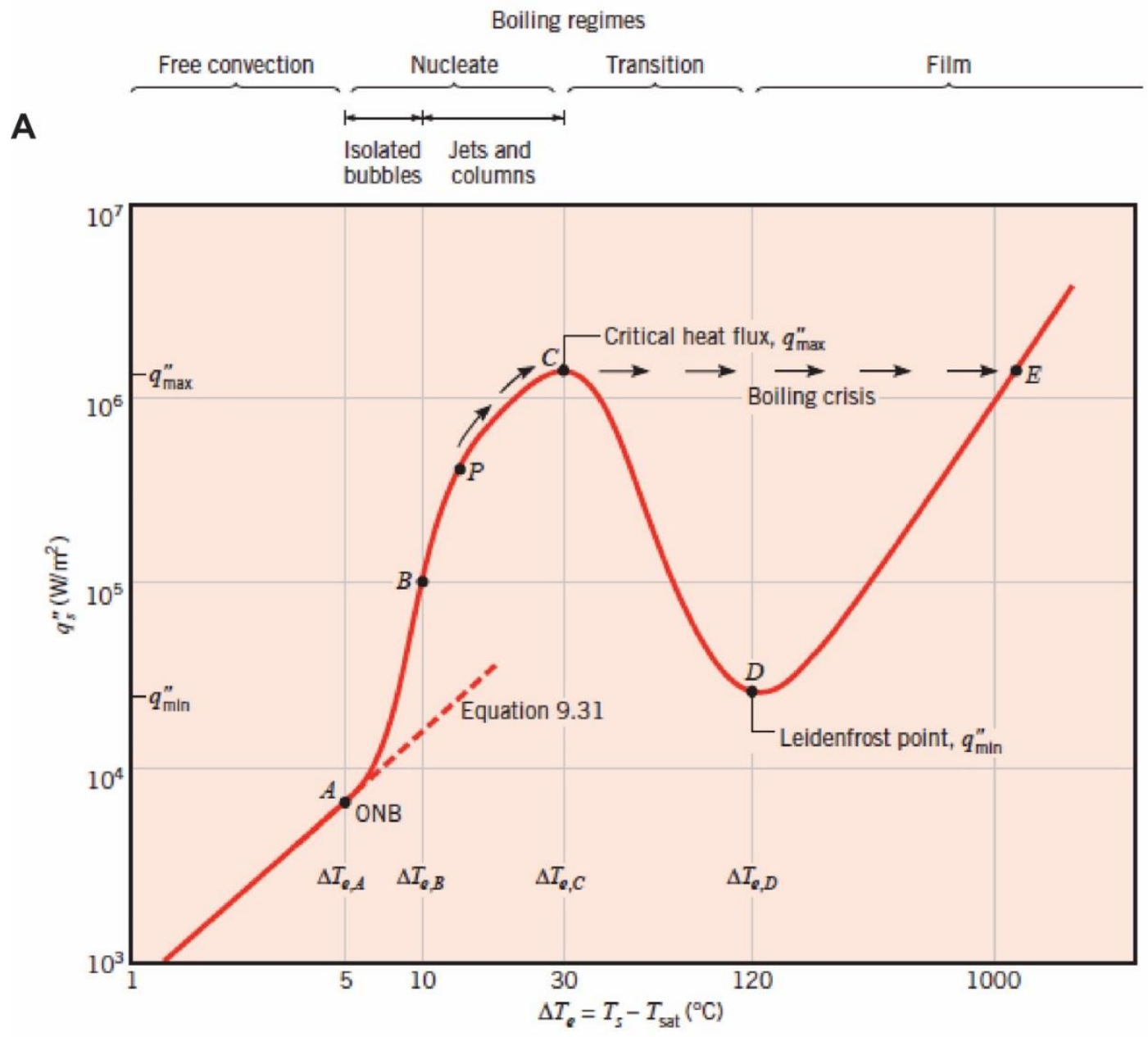

B

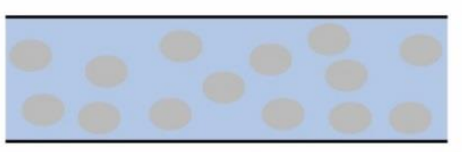

Bubble flow

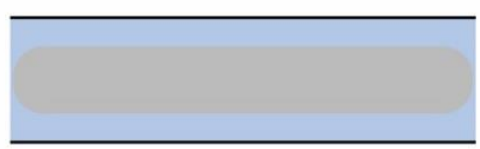

Slug-annular flow

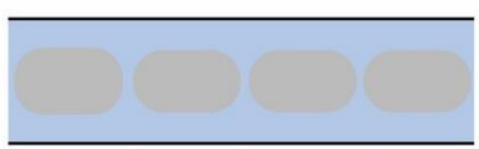

Slug flow

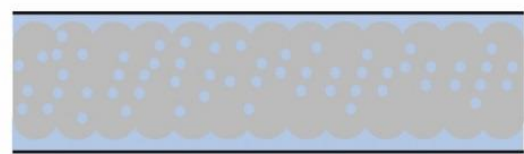

Annular flow

Figure 9. Boiling curve and regimes: (A) boiling curve obtained through a typical pool boiling experiments, reproduced by the permission of John Wiley and Sons (Bergman et al. 2006), B Different fluid regimes in boiling process.

When a liquid in contact with a substrate is heated by raising the solid temperature, initially the temperature rise causes natural convection. This is maintained up to a small degree of degree of superheat $(\Delta T)$, i.e. the solid temperature rising slightly above the liquid saturation temperature $\left(T_{s}\right)$. Eventually, the first stage of boiling is initiated and is 
manifested by nucleation and departure of bubbles from the solid surface. This is referred as onset of nucleate boiling (ONB) and it is characterized by a sharp change in the slop of heat flux (see the boiling curve in Figure 9A) (see also chapter 43, Nucleate Pool Boiling). The slope change shows the effectiveness of two-phase heat transfer over single phase and the change is also marked by a much higher heat transfer coefficient. Therefore, nucleate boiling sought after in many industrial processes. The bubble nucleation/departure also induces significant fluid motion, especially near to the solid surface. The bubble nucleation intensity depends on the degree of superheat $\Delta T$ and the availability of active nucleation sites on the solid surface. As the temperature of the solid surface increases ( $\Delta T$ increases), more nucleation sites become active, more bubbles nucleate, and coalesces after departing from the solid surface. With progressive rise in $\Delta T$, the boiling become more vigorous and starts producing high density of bubbles that eventually coalesces near to the solid surface to form a thin vapor film. This thin film add thermal resistance and also starts to obstruct the liquid from coming in contact with the solid surface. This is marked by a decrease in the gradient of the heat flux curve. At sufficient high $\Delta T$, the vapor covers the solid surface completely, preventing the liquid contact with the surface. This is called the dry-out or boiling crisis, and is marked by a sharp reduction in $q$, as shown in Figure 9A. The critical heat flux $(C H F)$ defines the maximum heat flux just before the dry-out. CHF is a surface specific characteristic, which depends on the surface textures and wettability. Different surface modification techniques are adopted to enhance CHF.

The decrease in q reaches a minimum at the Leidenfrost point, beyond which boiling progress with presence of a stable film on the solid surface. This regime, with much lower heat transfer coefficient compared to the nucleate boing is referred as film boiling (see also chapter 44, Transition and Film Boiling). Literature is rich with many empirical correlations to predict CHF and heat transfer coefficient in nucleate and film boiling regimes (Li and Peterson 2007; Kandlikar 2010). CHF and heat transfer coefficient are, largely, functions of physical properties of the liquid (viscosity, density, specific heat, thermal conductivity, and surface tension), bubble departure diameter, liquid flow rate, etc. (John G. Collier and John R.Thome 1994)

There are two modes of boiling: pool boiling and convective boiling.

1 In pool boiling, a heated surface is introduced in a pool of liquid (coolant). The motion of the liquid is largely driven by the buoyancy force. The whole liquid, except liquid near the solid surface, is below the solid surface temperature, $T_{s}$. Pool boiling is also called the subcooled boiling. 
2 In convective boiling, the liquid is forced by external means, as for example pump, to flow over the heat solid surface. The liquid near to the heated surface is rapidly replenished by the cooler liquid, and therefore, a higher heat transfer coefficient is achieved.

Boiling is used widely in industry for enhance heat transfer and thermal management applications across scale. Two most common exploitations in small scale are discussed below.

\subsubsection{Pool boiling on porous surfaces: effect of surface textures}

Boiling performance is greatly enhanced on micro- and nano-porous textures that provide a large area for heat transfer and enhance the liquid suction. Therefore, in the past few decades, different engineered micro- and nanoporous surfaces have widely been used to study boiling (Theofanous et al. 2002; Hsu and Chen 2012; Hetsroni et al. 2005). However, these experiments indicate that the design of micro- and nanoporous wick structure needs further improvement to enhance the boiling performance (Attinger et al. 2014). Therefore, there is a clear need to understand the nucleation and the subsequent boiling events on the porous matrices.

Typically, surfaces have pits and cavities, which can entrap gas or vapor leading to initiate the heterogeneous nucleation. The Gibbs free energy for creating a vapor nuclei on a smooth surface is given by (Collier 1972)

$$
G_{\text {het }}=\phi G_{\text {hom }}
$$

where $G_{\text {hom }}$ denotes the Gibbs free energy required to create a vapor nucleus in the bulk liquid, commonly referred as the homogeneous nucleation, and $\phi=\frac{2+2 \cos \theta+\cos \theta \sin ^{2} \theta}{4}$, with $\theta$ denoting the solid/liquid contact angle. For textured superhydrophobic surfaces, the superheat required for heterogeneous nucleation becomes small as $\phi$ exponentially goes to zero. Betz et al. (Betz et al. 2013) confirmed that indeed the heterogeneous nucleation occurs on textured superhydrophobic surfaces at a two orders of magnitude lower superheat compared to smooth surfaces. Therefore, it is clear that surfaces with low wettability towards the coolant liquid favor the nucleation. However, low wetting surfaces are not beneficial in later stage boiling, near to CHF. At CHF, the extended liquid layer on the heated surface ruptures, and vaporizes independently. Therefore, a wettable surface is needed to sustain the liquid film on the heater surface and, by doing so, a higher CHF can be obtained (Theofanous et al. 2002). These contradicting requirements make the fabrication of surfaces for optimal boiling performance challenging. Biphilic surfaces, with 
both hydrophobic and hydrophilic character, have been proposed for optimum boiling performance (Forrest et al. 2010; Hsu and Chen 2012).

Nanotextured surfaces offer a few unique features that can help overcome the surface wettability challenge. Such texturing can help tune the wettability to extremes. For example, it has been shown shown that a nanotextured superhydrophillic surface can not only facilitate the liquid transport to the heated area by its superwettability (Takata et al. 2005) and enhanced capillary transport (R. Chen et al. 2009), but also provide the enhance surface area ( $\mathrm{Li}$ and Peterson 2007) for better heat transfer. With the advancement of sophisticated micro- and nano-fabrication techniques, and their characterization proficiency, different nanotextures (Lu et al. 2011; Yao et al. 2011; Dai et al. 2013; R. Chen et al. 2009; Li and Peterson 2007) were fabricated and their potential to significantly enhance boiling heat transfer performance has also been established. For example, a $100 \%$ enhanced CHF was achieved with a silicon nanowire forest compared to a smooth silicon surface (Lu et al. 2011). A sustained operation under high temperature and need to optimize the surface thermal resistance are some outstanding challenges which need to overcome before nanotextured surfaces could be widely adopted in practical applications. Additionally, there also is a need for theoretical studies to complement the experimental findings discussed above.

\subsubsection{Convective boiling: microchannel flow boiling}

An enhanced heat transfer performance is achieved by adopting convective boiling, where the liquid is forced by external means (e.g. a pump). Such convective or flow boiling in microscale devise frequently occurs with liquid flowing through a microchannel (see chapter 48, Flow Boiling in Tubes for macroscale boiling phenomena). The configuration also provides a convenient means to understand the basic principle, thus our discussion will be focused on this. It is pertinent to start by introducing the two dimensionless numbers.

First, Bond number (Cheng et al. 2007) is the ratio of gravitational to surface tension forces and can be expressed as

$$
B o=\frac{g\left(\rho_{l}-\rho_{v}\right) D^{2}}{\gamma_{l-v}}
$$

where $g$ and $\rho_{v}$, respectively, denote the acceleration due to gravity and the density of the vapor phase. With small size $(D)$, in microchannels the $B o$ is small relative to minichannel and macrochannel flow boiling. Therefore, in microchannel flow boiling surface tension effects dominates over the gravity.

Second, the boiling number (Wang et al. 2007) is the ratio of heat flux to the mass flux and can be expressed as 


$$
B o_{i}=\frac{q}{G h_{f g}}
$$

where $G$ and $h_{f g}$ denote mass flux and latent heat of evaporation, respectively. $B o_{i}$ helps to define the stability of different flow boiling regimes that follow.

The flow boiling regimes are classified according to the nature of vapor and liquid phases (Cheng et al. 2007; Wang et al. 2007) and are accordingly termed bubble flow, slug flow, slug-annular flow, and annular flow (see Figure 9B). These regimes are observed at progressively higher heat fluxes. The liquid acts as the continuous phase and vapor as the dispersed phase. With the constant flow of the liquid, and at a low heat flux conditions, small bubbles are dispersed into the liquid continuum. This flow pattern is called bubbly flow (Cheng et al. 2007; Wang et al. 2007). At a slightly higher heat flux, bubbles get bigger and the flow regime turns to slug flow. With increase in heat flux, the bubble generation frequency increases and the bubbles start to coalesce and form a continuous vapor stream. This is referred as the slug-ring (or slug-annular) flow. With even greater heat flux, the vapor starts to occupy the channel core and a thin liquid film surrounds the vapor core. Interestingly, miniature liquid droplets are still entrained in the vapor core. This regime is called annular flow.

All the above flow regimes have been observed in microchannel flow boiling. The stability of flow regime depends on the mass and heat flux (Wang et al. 2007; Cheng et al. 2007). When $\mathrm{Bo}_{\mathrm{i}}$ is less than 0.96, a stable flow regime is observed and is characterized by bubble generation at the channel wall and followed by their steady transportation along the channel width. The long oscillation, and unstable flow regime are observed when $0.96<\mathrm{Bo}_{\mathrm{i}}<2.14$. This is marked by temperature and pressure variations and switching between bubbly and annular flows. Short oscillations with unstable flow regime is observed with $\mathrm{Bo}_{\mathrm{i}}>2.14$ (Kandlikar 2010; Wang et al. 2007) and is characterized by short periods of oscillations featuring periodic dry out and rewetting. With further increase in the heat flux increases, the annular flow becomes stable and the vapor quality increases at the outlet of the microchannel leading to poorer heat transfer performance (Qu and Mudawar 2003; Hetsroni et al. 2005). Interestingly, in the annular flow regime, channel wall and inlet liquid temperature increase gradually. The resulting heat transfer performance at different vapor qualities are not well predicted by the existing models (Lee and Mudawar 2005), which clearly reflects a need for deeper theoretical studies on flow boiling in microchannels. 


\subsubsection{Micro/nanotextured surfaces for efficient condensation heat transfer}

Vapor condensation occurs when the environmental temperature is below the saturation temperature of the vapor. During condensation, the latent heat (enthalpy of vaporization) is released. This is used in many processes, such as air conditioning systems, in refrigeration, in generation of electric power, etc. Most of these applications involve condensation solid surfaces maintained below the saturation temperature. The difference between saturation temperature and the solid surface temperature is referred as degree of subcooling. Depending on the surface texture and surface chemistry, the liquid condenses either in the form of droplets or a film, referred respectively as dropwise and filmwise condensation (see also chapter 52 on Film and Dropwise Condensation). Both droplets and the liquid film (condensate) prevent the contact of the vapor phase with the solid and increase the resistance to the heat transfer. Typically, a higher - even up to an order of magnitude - heat transfer coefficient is achieved in the dropwise condensation compared to the filmwise condensation. Therefore, realizing surfaces that sustain stable dropwise condensation is highly desirable. Regardless of the nature of condensation, similar to the boiling phenomena, condensation proceeds through two steps- nucleation of the condensate, and the growth of this condensate either to larger drops or a liquid film. In case of dropwise condensation, nucleation, growth and the subsequent departure of the condensate droplets exposes new areas on the solid surface, for cycle to continue. Therefore, a surface offering many nucleation sites and small roll-off angle for droplet departure (i.e. low adhesion between drops and the solid surface) should be ideal to enhance condensation heat transfer.

The Gibbs free energy for heterogeneous nucleation of condensate can be expressed by the same equation 1.35 as discussed in the boiling section above. The factor $\phi$, however, has the opposite trend than that of boiling; thus, hydrophilic surfaces are better at facilitating nucleation compared to hydrophobic surfaces. However, hydrophilic surfaces promote formation of a liquid condensate film instead of droplets, which affects the efficiency of thermal transport. Droplet adhesion on the other hand is lower on hydrophobic surfaces. Thus, hydrophilicity is needed for nucleation of liquid condensate, whereas hydrophobicity is required to maintain a dropwise condensation (Attinger et al. 2014). Clearly, there is a need for a trade-off to optimize the rate of nucleation, drop adhesion and stabilize dropwise condensation, without worsening the surface thermal resistance. Advancement of micro-/nano-fabrication, and wettability engineering offer some exciting opportunities and are discussed next. 
The size of the departing droplet is crucial in the condensation heat transfer. The heat transfer coefficient decreases as the maximum droplet departure size increases (Fevre and Rose 1966, 1965). A number of studies have focused on passively controlling the drop radius, mobility and size distribution by engineering the condensing surface and/or tailoring surface chemistry. Firstly, applying the surface energy gradient (Daniel et al. 2001; Macner et al. 2014) is an effective way to promote mobility. In fact, surfaces with patterned with hydrophobic and hydrophilic regions, can be used to optimize the drop nucleation and mobility. Studies have already established the feasibility of controlling maximum departing droplet diameter, and its mobility by tuning the width of hydrophobic and hydrophilic regions (Chatterjee et al. 2013; Chatterjee et al. 2014; Peng et al. 2015). Secondly, on grooved superhydrophobic surfaces, condensate droplets coalesce and can be easily drained through grooves, leaving behind the top portion of surfaces dry due to its superhydrophobic character (Narhe and Beysens 2004; Izumi et al. 2004). Notwithstanding the success of these approaches in demonstrating dropwise condensation, these surface fabrication technologies have been realized only on small samples (surface dimension millimeter to centimeter) and, more significantly, they were evaluated through condensation experiments lasting a few hours or less, which raises questions regarding their industrial exploitation. There also are significant challenges towards scalability of fabrication (Attinger et al. 2014). To this end, robust and rationally conceived surface treatment strategies facilitating stable dropwise condensation are being actively researched. Simple, thick polytetrafluoroethylene (PTFE) coatings have demonstrated stable dropwise condensation without aging for $\sim 1000$ hours (Rose 1997; Ma et al. 2000). However, the PTFE coatings (especially at a high thickness) introduce substantial thermal resistance, thereby negating the efficiency gained due to dropwise condensation. Applying self-assembled monolayer (SAM) of silanes is a way to overcome the thermal resistance issue as the SAMs have lower thickness ( $\sim 30 \mathrm{~A})$; durability however is an issue in this case. Interestingly, a recent work has shown that a graphene coating can sustain dropwise condensation over two weeks on continuous exposure to steam (Preston et al. 2015). Additionally, due to their higher thermal conductivity, the graphene coating also showed a fourfold higher heat transfer coefficient compared to a silane based coating. Recently, Paxson et al. (Paxson et al. 2014) synthesized metal grafted copolymer thin films via Initiated Chemical Vapor Deposition (iCVD) technique. Those metal grafted thin films showed stable dropwise condensation without aging up to $\sim 50$ hours, with a good heat transfer performance. 
A

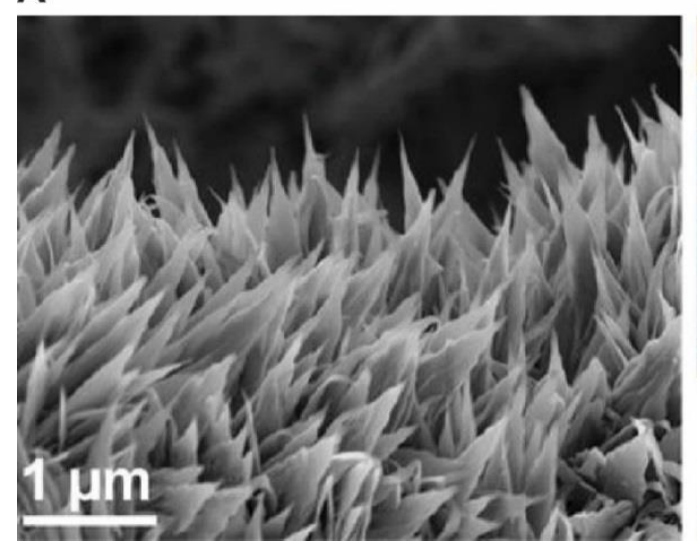

B

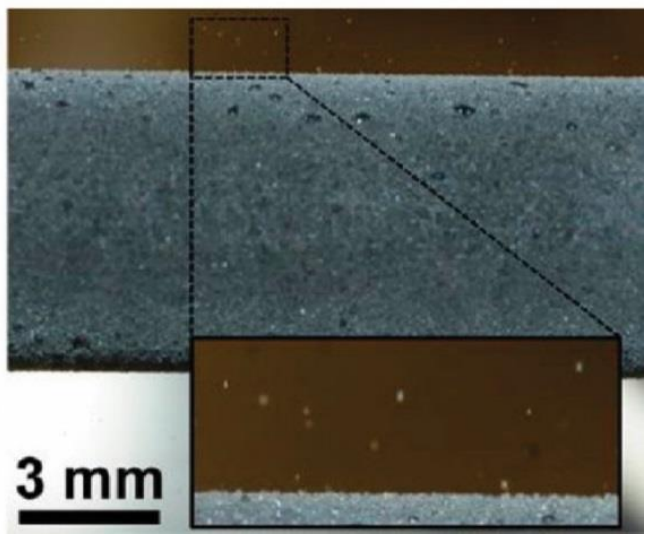

Figure 10. Jumping drop condensation: (A) SEM image of copper based nanotextured superhydrophobic surface, (B) Photographic image of dropwise condensation on a nanotextured copper pipe. The inset clearly shows coalescing droplets jumping off the surface, which was shown to facilitate high efficiency of condensation heat transfer. Reproduced by the permission of American Chemical Society (Miljkovic et al. 2013).

As an exciting finding, at low surface subcooling, it has been shown that condensate drops coalescing on nanotextured superhydrophobic surfaces can spontaneous jump from the surface due to release excess surface energy (see Figure 10, Boreyko et al. 2011; Chen et al. 2007; Miljkovic et al. 2013). The size of maximum departing droplet is reduced and self-regulated by this jumping droplet phenomena. This results in a stable dropwise condensation and an improvement in heat transfer performance by $25 \%$. Moreover, this spontaneous jumping behavior can also be augmented by application of an external electric field (Miljkovic et al. 2013).

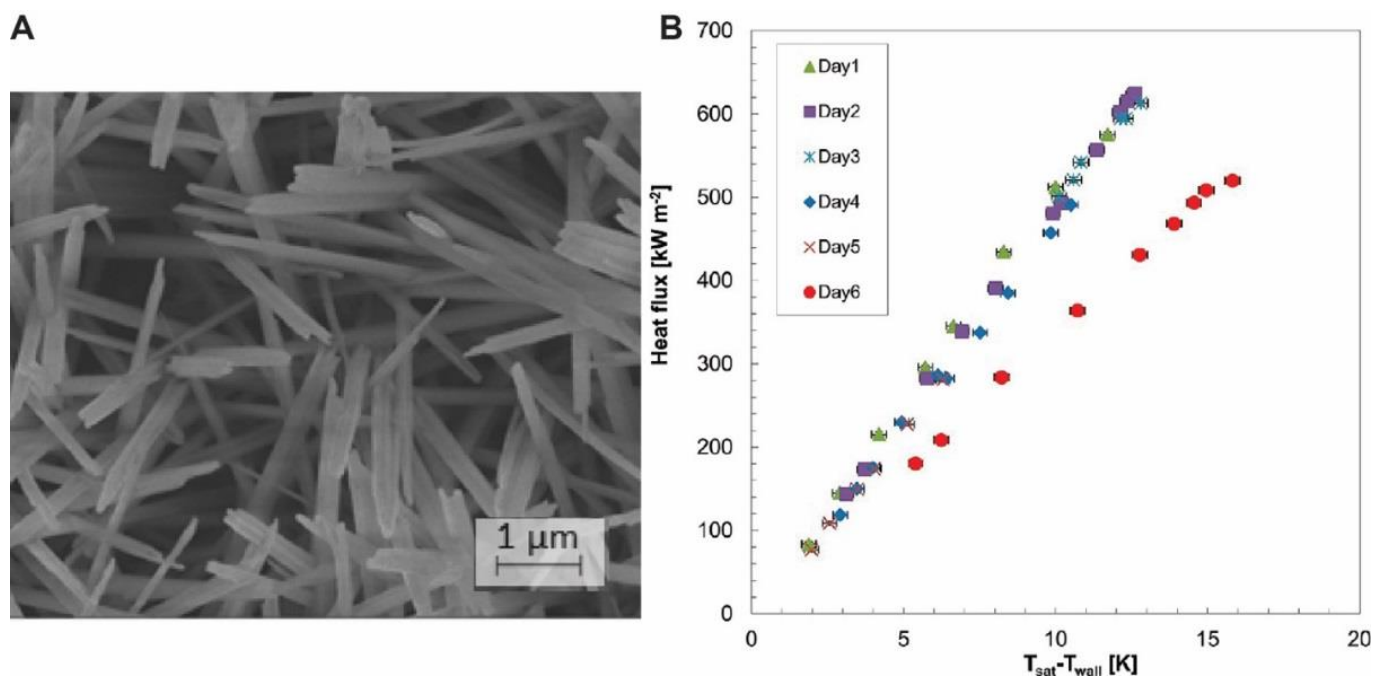

Figure 11. A stable film condensation on copper base nanotextured surfaces: (A) SEM image of the copper based nanotextured surface, and $B$ The plot of heat fluxes obtained on such surfaces as a function of the degree of subcooling over six consecutive days 
of condensation experiments at $110^{\circ} \mathrm{C}$ and $12 \mathrm{~m} / \mathrm{s}$ vapor shear flow. Reproduced by the permission of American Chemical Society (Torresin et al. 2013).

Most of the above mentioned condensation experiments testing surface treatment were performed in a customized closed vessel at low pressure conditions and at low temperature $\left(<100{ }^{\circ} \mathrm{C}\right)$. In most industrial applications, steam condensation occurs in even more stringent conditions. For example, in an electric power plant (Beér 2007), steam condensation occurs in presence of vapor shear flow. Therefore, the synthesis of a hydrophobic coating, which is durable at such conditions, is a formidable problem. To this end, Torresin et al. (2013) performed condensation experiments on copper based nanotextured surfaces at a high temperature $\left(\sim 110^{\circ} \mathrm{C}\right)$ and in the presence of vapor shear speed reaching as high as $\sim 18 \mathrm{~m} / \mathrm{s}$. They used a scalable wet chemistry based etching to generate the surface nanotexture. A representative morphology of the surface is shown in the SEM image in Figure 11A. Surface texturing was followed by applying a perfluorinated thiol SAM to achieve superhydrophobicity. The nanotextured surfaces were evaluated in a high temperature flow condensation condition for six consecutive days. Figure 11B shows the plot of experimentally measured heat fluxes on this surface as a function of the degree of subcooling, at a vapor shear speed of $12 \mathrm{~m} / \mathrm{s}$. Impressively, a high and stable heat transfer coefficient, derived from the slope of the curves in the Figure 11B, was maintained up to five consecutive days of tests. The work established a proof of concept for stable micro/nanoengineered surfaces for flow condensation; however, further work is necessary to improve the surface robustness for industrial exploitation.

\subsubsection{Nanoengineered icing}

Ice formation on solid surfaces is one of the commonest phase change problems encountered in everyday life. Controlling ice nucleation on a surface is an important challenge. Thus, whereas in food products such as ice creams it is desirable to ensure a certain density of ice nuclei, whereas ice formation is unwanted in a number of application such as on infrastructure components, aerospace, marine, automotive and built environment because it can pose a serious challenge to human safety. In particular, the progress in superhydrophobic surface manufacture has led to renewed interests in design of 'icephobic' surfaces that can delay ice formation, resist impact of supercooled drops and/or facilitate easy removal of ice from the surface with a low surface-ice adhesion(Tourkine et al. 2009; Kulinich et al. 2011; Maitra et al. 2015). To this end, micro/nanoengineered surfaces offer good promise, though a careful 
control of surface texture, surface chemistry, environmental parameters, such as temperature and relative humidity, etc. is important to facilitate extraordinary icephobicity. The following sections outline these different facets in brief. Dedicated reviews should be consulted for a more detailed discussion (Schutzius et al. 2014; Kreder et al. 2016; Nath et al. 2016).

\subsubsection{Ice nucleation: towards ice-free surfaces}

A liquid can reach below freezing temperature and exist in a metastable, supercooled state. Ice clusters/germs form continuously in such a liquid. However, the clusters must reach to a critical radius for initiation of spontaneous freezing. The Gibbs free energy associated to form an ice cluster $\left(\Delta G_{c l}\right)$ is the sum of Gibbs free enrgy to make a new interface $\left(\Delta G_{s}\right)$ and the volumetric Gibbs free energy associated with forming bonds of between molecules $\left(\Delta G_{v}\right)$,

$$
\Delta G_{c l}=\Delta G_{s}+\Delta G_{v}
$$

For a spherical cluster containing $i$ molecules, , denoting the cluster radius with $r_{i}$, the above equation can be converted to (Murray et al. 2012)

$$
\Delta G_{c l}=-\frac{4 \pi r_{i}^{3}}{3 v^{\prime \prime}} k T \ln S+4 \pi r_{i}^{2} \gamma_{I W}
$$

where $k$ is the Boltzmann constant, $\gamma_{I W}$ ice-water interfacial energy, $v$ "is the molecular volume of ice, and $S$ is the ratio of the vapor pressure of liquid water and ice.

Equation 1.39 can be used to determine the critical ice nucleus radius $\left(r_{c}\right)$ to initiate the freezing process by equating $\frac{d \Delta G_{c l}}{d r_{i}}=0 ;$ the result is

$$
r_{c}=\frac{2 v^{\prime \prime} \gamma_{I W}}{k T l n S}
$$

Using standard property tables, at $-20{ }^{\circ} \mathrm{C}, r_{c} \approx 2 \mathrm{~nm}$. The expression in equation 1.40 shows the critical ice cluster radius for homogeneous ice nucleation. By substituting back in equation 1. 39 the critical free energy barrier for nucleation can be obtained as

$$
\Delta G_{C}^{h o m}=\frac{16 \pi \gamma_{I W}^{3}}{3}\left(\frac{v^{\prime \prime}}{k T \ln S}\right)^{2}
$$


For ice nucleating on a solid surface (heterogeneous nucleation), it can be shown that

$$
\Delta G_{C}^{h e t}=\frac{16 \pi \gamma_{I W}^{3}}{3}\left(\frac{v^{\prime \prime}}{k T \ln S}\right)^{2} f\left(\theta_{I W}, \mathrm{R}^{\prime}\right)
$$

The factor $f\left(\theta_{I W}, \mathrm{R}^{\prime}\right)$ on the right hand side is a function of the contact angle of the ice cluster on the solid surface and surrounded by the supercooled water and the characteristic local surface roughness radius of the solid $\left(R^{\prime}\right)$ (Fletcher 1958). The above expressions (equations 1. 40 to 1.42) are the results from the so called classical nucleation theory. Generally, all natural surfaces have nanopits and bumps, and particularly, pits enhance the ice nucleation. For $R^{\prime}$ is comparable to $r_{c}$, the factor $f$ is less than 1 , thus the heterogeneous nucleation becomes favorable. For example, for $\theta_{I W}<90^{\circ}$, a concave surface nanopit with radius comparable to the $r_{c}$ will enhance ice nucleation probability and vice versa. This broad conclusion from classic nucleation theory was however recently shown to be inaccurate by Eberle et al. (Eberle et al. 2014). With careful experimentation they showed that ice nucleation was insensitive to roughness and argued that this was due to presence of a quasi-liquid layer (QLL) at the interface between the solid and ice embryo. QLL is an experimentally observed fact and (Engemann et al. 2004; Howells et al. 2005) was also shown recently to affect ice adhesion on surfaces (Chen et al. 2017).

\subsubsection{Effect on environmental conditions on ice nucleation}

Environmental conditions like relative humidity, gas flow are crucial to determine ice nucleation mechanism(Jung et al. 2012a). Particularly, in an unsaturated environment in presence of gas flow, the temperature of droplet/air interface decreases due to evaporative cooling, as shown in Figure 12A. Jung et al. 2012 (Jung et al. 2012a) studied this using droplet sitting on various surfaces in presence of air flow, under different ambient humidity. On a superhydrophobic surface, they observed that ice nucleation initiates from droplet/air interface (homogenous nucleation) instead of commonly expected heterogeneous nucleation where ice nucleation initiates from droplet/surface interface. The effect is important because it can potentially take out the effect of substrates (heterogeneous nucleation) from freezing. The switch in nucleation mechanism and temperature change in the droplet can be explained by considering heat transfer physics as described below. 

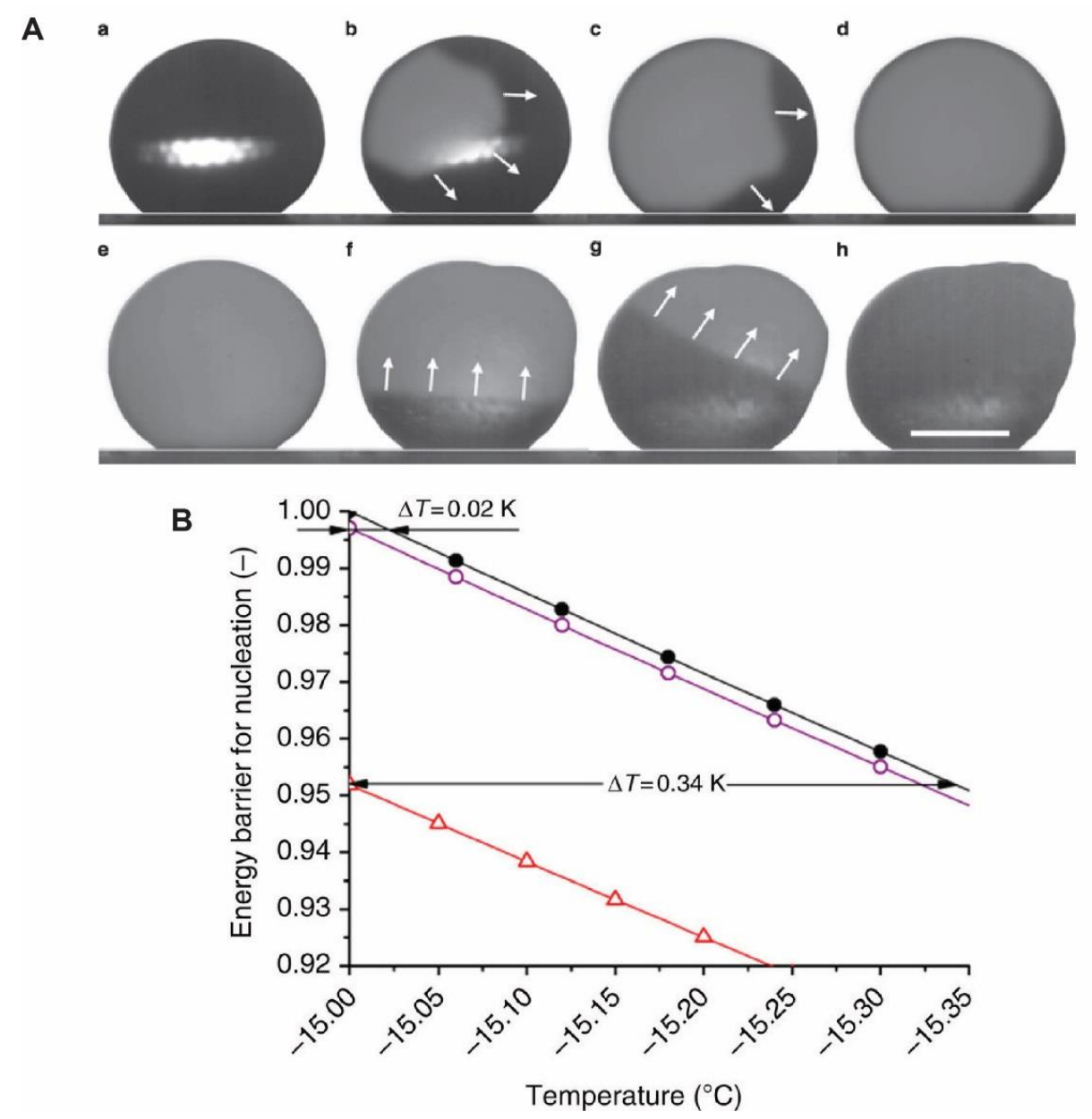

Figure 12. Ice nucleation mechanism in presence of shear flow: (A) Snapshots of drop freezing on a superhydrophobic surface, capturing the two stages of freezing. (a)-(d) First, recalescent freezing starting with homogeneous nucleation at the droplet/gas interface. (e)-(h) Second stage of freezing starting from drop/solid interface. Note the second stage is inevitable after the first, thus the first one is controlling. (B) Plot of free energy barrier for homogenous and heterogeneous nucleation as a function of temperature. Filled circles represent the homogeneous nucleation, and open circles represents heterogeneous nucleation for the superhydrophobic surface. Open triangles represent the nucleation on a hypothetical surface with contact angle of $130^{\circ}$ where homogeneous nucleation should switch back to heterogeneous nucleation even in presence of gas flow. Reproduced by the permission of Nature Publishing Group (Jung et al. 2012)

Considering an evaporating droplet, with the substrate at same temperature as the surrounding, the energy used for water evaporation at vapor/liquid interface must be equal to energy received by droplet from the surrounding gas and by heat conduction from bottom of the droplet to the top (Jung et al. 2012a). Thus the energy balance becomes,

$$
\begin{gathered}
\dot{Q_{v}}=\dot{Q_{g}}+\dot{Q_{w}} \\
\dot{Q}_{v}=\Delta H_{v} h_{f g} \dot{n} \\
\dot{Q}_{v}=h_{g} A_{g w} \dot{n} \Delta T
\end{gathered}
$$




$$
\dot{Q_{w}}=k_{w} \frac{A_{s w}}{l} \Delta T
$$

where $\Delta H_{v}, \dot{n}, h_{g}, k_{w}, l$, and $A$ denote the molar enthalpy molar evaporation rate of water, heat transfer coefficient, thermal conductivity, maximum droplet height, and interfacial area, respectively. $\Delta T$ denotes the tempeture difference between the vapor/liquid interface and the surrounding (the substrate and surrounding has were in thermal equilibrium). The subscripts $g, v$, and $w$ denote gas, vapor, and water phase, respectively. From equation $1.46, \Delta T$ can be estimated with the knowledge of $h_{g}$ and $\dot{n}$. Jung et al. considered a correlation for determining $h_{g}$ with flow and standard analytical result for the case of pure diffusion (no flow).

The molar $\dot{n}$ can be written as follows

$$
\dot{n}=\frac{\rho_{w}}{M} \dot{v}=\frac{\rho_{w}}{M} \frac{A_{g w}}{r_{o}} \xi
$$

where $\dot{v}, M, r_{o}$ and $\xi$ denote volumetric water evaporation rate, molecular weight of water, initial radius of water droplet and the evaporation rate constant, respectively. Thus, by combining equations 1.43 to 1.46 ,

$$
\Delta T=-\frac{\Delta H_{v} \xi \rho_{w}}{M r_{o}} \frac{1}{\left(h_{g}+\frac{k_{w}}{l}\right)}
$$

The evaporation rate constant $\xi$ can be determined from experiments (Jung et al. 2012a). With the knowledge of $\xi$ and $h_{g}$, at the surrounding temperature of $-15{ }^{\circ} \mathrm{C}$ and humidity of $30 \%, \Delta T$ for forced convection and pure diffusion (in absence of flow) turned out to be $0.34 \mathrm{~K}$, and $0.02 \mathrm{~K}$, respectively. Therefore, in presence of external flow, the reduction in temperature at the liquid/vapor interface is clearly indicated the influence of convection. The free energy barrier expressions in equations 1.41 and 1.42 can be used to obtain energy barriers as function of the contact angle. Jung et al. plotted these for a superhydrophobic surface in Figure (12A). Their plot as a function of $T_{w}$, reproduced in Figure $12 \mathrm{~B}$, shows the variation of free energy barriers at two different substrate wettabilities. The energy barrier for homogeneous nucleation is smaller compared to heterogeneous nucleation in presence of gas flow (with associated temperature reduction at the drop/vapor interface). This is a vital conclusion, which indicates that in presence of gas flow ice nucleation initiates from droplet/gas interface, not from droplet/surface interface, and therefore, there is role of surface engineering on ice nucleation mechanism. 

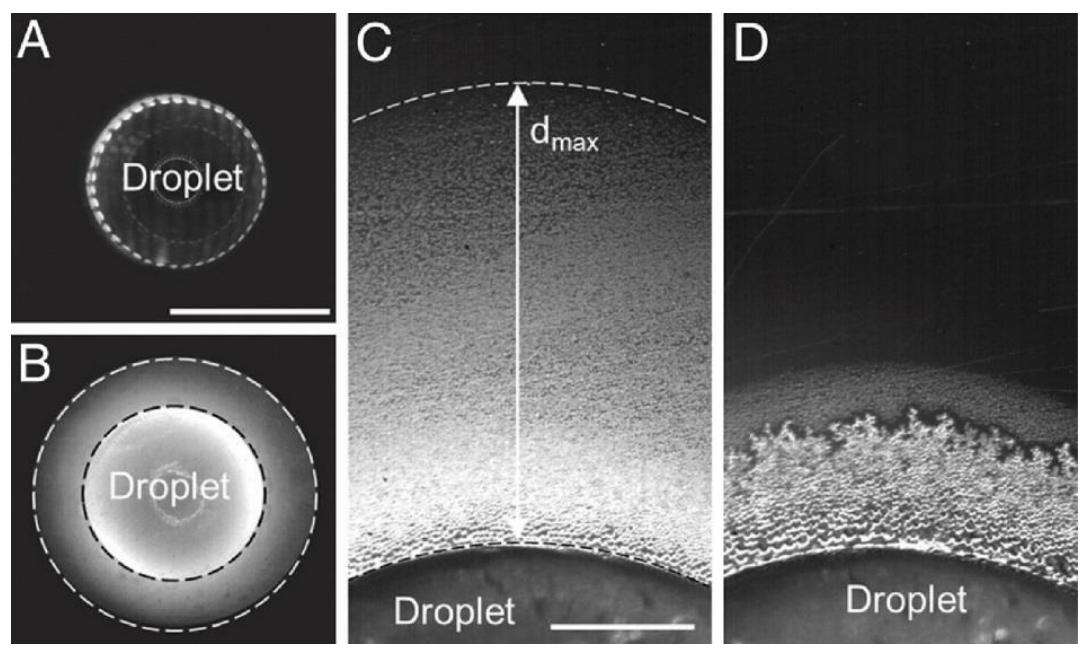

Figure 13. Evaporation and condensation of water droplet on a polymeric substrate. (A) top view optical microscope image of a supercooled water droplet, (B) a condensation halo formation (white dashed circle) around the droplet (black dashed circle), (C) a magnified view of the condensation halo around the droplet. $D_{\max }$ indicates maximum expansion of the condensation halo. (D) Partial freezing of the condensate during its evaporation. Scale bars in A and Bare $2 \mathrm{~mm}$, and in C and D are $400 \mu \mathrm{m}$. Reproduced by the permission of Proceeding National Academy of Science of the United States of America (Jung et al. 2012).

The group also investigated the role of environmental factors and in particular substrate thermal conductivity on frost formation. Frosting significantly affects the ice nucleation. Although common in humid environments, frosting can also occur during the freezing of droplets. Typically, droplets freeze through two overlapping stages - the first stage is the recalescent freezing, and it is followed by a second isothermal freezing stage. For a droplet freezing in an unsaturated environment (see Figure 13), the (latent) heat released by freezing can cause a significant evaporation of the remaining liquid. The vapor from this the evaporation, condenses on the surface leading to a formation of a condensation halo around the droplet (as shown in Figure 13A and B). The vapor generated condensed around the original droplet due to low surrounding temperature, thereby forming a ring of condensates. The amount of evaporation and hence the size of the condensate ring was greater on a thermally insulating (polymeric) substrate, compared to a conductive copper one. This is because the heat released by freezing was unable to escape as efficiently on the insulating substrate, leading to greater amount of evaporation. Part of the condensate around the droplet froze leading to formation of the frozen ring; on copper, however, the condensate could evaporate back into the surrounding, leaving no such frozen ring (Jung et al. 2012b). Interestingly, the authors also showed that the frozen condensation ring (halo) could initiate the freezing of a neighboring droplet. These results offer an insight into how an array pf droplets on surface could freezing sequentially with freezing a droplet initiating freezing of its neighbors(Jung et al. 
2012b). Two key conclusions can be drawn based on this. Firstly for designing icephobic surfaces, thermally conductive substrates will be better at resisting such frost formation. Secondly, freezing not only depends on the substrate surface (wetting) properties but the substrate thermal characteristics and the environmental conditions. To this end, the frost formation is critical in surface icing not only because the frost can initiate the freezing of the adjacent supercooled droplets also frost can significantly increase ice adhesion.

\section{Summary and future trend}

This chapter has focused on presenting the basic facets of heat transfer physics at small scales (micro- and nano-scales) in order to help contextualize the active current research in micro/nanoscale thermal devices. The following summarizes the chapter and outlines the authors' perspective on the scope and the need for future research.

\section{Conduction at microscale: Thermoelectricity and Thermal Interface Materials}

Thermoelectric performances of superlattices, metal oxide nanowires, organic polymers etc. have been studied extensively. These materials were engineered at nanoscale to improve ZT values by reducing their thermal conductivities, without significantly altering $\sigma$ and $S$. Till date, a highest ZT value of 2.5 was obtained with $\mathrm{Bi}_{2} \mathrm{Te}_{3} / \mathrm{Sb}_{2} \mathrm{Te}_{3}$ superlattices (Venkatasubramanian et al. 2001). New nanostructured materials have the exciting potential to achieve similar performance while exploiting abundant materials. To this end use of Si and Ge based nanostructures was discussed in detail. Recent research has also raised the potential of metal-chalcogenide based materials as alternate cheap thermoelectrics (Han et al. 2016). Scalability of fabrication, long term usage and performance reliability are largely open questions for nearly all nanostructured thermoelectrics.

Future applications of thermoelectric materials may not be limited to energy harvesting and thermal management. Exciting opportunities are arising in biomedical industry, for example, implantable medical devices (IMDs) (Paulo and Gaspar 2010; Hannan et al. 2014) such as pacemakers, defibrillators, infusion pumps etc. IMDs require a constant source of energy for their operation, typically supplied through batteries. Potentially, thermoelectric materials can be implemented with IMDs since they can capture energy from the body itself (Paulo and Gaspar 2010). However, such possibilities will be contingent on the development of biocompatible thermoelectric materials.

Emerging nanostructured materials were discussed for thermal interfaces such CNT and graphene polymer nanocomposite and vertically aligned CNT forests. Despite great potential, the performance of CNT and graphene nanocomposites suffers from high thermal interfacial resistance; strategies such as modifying surface chemistry offer 
potential room for (significant) improvement. Additionally, CNTs/graphene can be grown on both the interfacing substrates to eliminate the inherent acoustic mismatch between substrate and CNT/graphene.

\section{Single phase convection}

Miniaturized modern electronic devices produce an enormous heat fluxes that limits their reliable operation. Moreover, the heat generation problem is becoming more challenging due to upcoming 3D IC designs. To date MMC heat sinks using water as coolant have been shown to address current thermal needs with good trade off against coolant pumping power. Interesting finding on using micropins as cooling structures in heat sinks is also worth exploring further. For example, due to microscale confinement vortex shedding from micropins occurs at $R e \sim 800$ leading to a uniform temperature distribution and a two-fold enhancement of heat transfer performances. Future studies need to focus on clarifying the effect of microscale geometry of the microchannel and the roughness of the channel walls on the cooling performance (Hassan et al. 2004).

\section{Phase change processes and heat transfer}

Broadly, three phase change processes, boiling, condensation and icing (liquid to solid), were discussed. The two phase heat transfer processes are lot more efficient because they are associated with release or absorption of latent heat. During phase change one phase is continuous - typically in a metastable state - and the other is discrete. For example, in boiling liquid (vapor), in condensation vapor (liquid), and in icing liquid (solid), respectively, is continuous (discrete) phase. The efficiency of phase change processes relies on the control of generation of the discrete phase, which can be achieved by altering surface chemistry and roughness at micro- and nanoscale. In fact, in the last few decades, a tremendous enhancement of phase change heat transfer processes was observed due to the development of micro/nano-fabrication techniques that enables us to control the surface roughness and chemistry. However, the understanding of the role of surface functionalities on the two phase processes remains elusive. The multiscale transient behavior of phase change processes makes the related theoretical studies difficult, and computational studies (computationally) expensive (Dhir et al. 2005; Attinger et al. 2014). This leaves clear room for future work.

Hierarchical (micro/nano) surfaces also offer additional benefits in two phase processes; for example, by offering greater mobility of liquid drops on hierarchical superhydrophobic surface. However, scaling the fabrication is a challenge and continues to motivate a number of excellent research articles. Furthermore, the surfaces need to be tested in more realistic conditions. For example, for condensation, flow condensation is industrially more relevant (Torresin et al. 2013), albeit challenging to sustain. Replication of realistic conditions in a standard laboratory 
environment also poses some challenges. For example, in icing, achieving a low supercooling state is difficult due to heterogeneous freezing; to date limited to $\sim-18^{\circ} \mathrm{C}$ for millimeter size drops (Maitra et al. 2014). This is in contrast to water drops at a temperature of $-40^{\circ} \mathrm{C}$ encountered by planes crossing clouds (Maitra et al. 2014). Future studies will need to consider these challenges to realize the exciting promise of nanostructured icephobic surfaces, while also considering the issue of surface durability.

\section{Acknowledgements}

TM acknowledges the funding from Swiss National Science Foundation (P2EZP2_162277) and MKT from ERC Starting Grant: NICEDROPS.

\section{Bibliography}

Alfieri F, Tiwari MK, Zinovik I, et al (2010) 3D Integrated Water Cooling of a Composite Multilayer Stack of Chips. J Heat Transfer 132:121402. doi: 10.1115/1.4002287

Asheghi M, Kurabayashi K, Kasnavi R, Goodson KE (2002) Thermal conduction in doped single-crystal silicon films. J Appl Phys 91:5079-5088. doi: 10.1063/1.1458057

Attinger D, Frankiewicz C, Betz AR, et al (2014) Surface engineering for phase change heat transfer: A review. MRS Energy Sustain 1:E4. doi: 10.1557/mre.2014.9

Balandin AA (2011) Thermal properties of graphene and nanostructured carbon materials. Nat Publ Gr. doi: 10.1038/NMAT3064

Balandin AA, Nika DL (2012) Phononics in low-dimensional materials. Mater Today 15:266-275. doi: $10.1016 / \mathrm{S} 1369-7021(12) 70117-7$

Balasubramanian G, Puri IK, Böhm MC, Leroy F (2011) Thermal conductivity reduction through isotope substitution in nanomaterials: predictions from an analytical classical model and nonequilibrium molecular dynamics simulations. Nanoscale 3:3714. doi: 10.1039/c1nr10421g

Beér JM (2007) High efficiency electric power generation: The environmental role. Prog Energy Combust Sci 33:107-134. doi: 10.1016/j.pecs.2006.08.002

Bergman TL, Lavine AS, Incropera FP, DeWitt DP (2006) Fundamentals of Heat and Mass Transfer, Sixth. John Wiley \& Sons, Inc.

Betz AR, Jenkins J, Kim C-J “CJ”, Attinger D (2013) Boiling heat transfer on superhydrophilic, superhydrophobic, 
and superbiphilic surfaces. Int J Heat Mass Transf 57:733-741. doi: 10.1016/j.ijheatmasstransfer.2012.10.080 Birctt F (1952) ELASTICITY AND CONSTITUTION OF THE EARTH’S INTERIOR*.

Bird RB, Stewart WE, Lightfoot EN (1966) Transport Phenomena. John Wiley \& Sons, Inc.

Bissessur R, Kanatzidis MG, Schindler JL, et al (1993) Encapsulation of polymers into MoS2 and metal to insulator transition in metastable MoS2. J Chem Soc Chem Commun 4:1582. doi: 10.1039/c39930001582

Boreyko JB, Zhao Y, Chen C-H (2011) Planar jumping-drop thermal diodes. Appl Phys Lett 99:234105. doi: $10.1063 / 1.3666818$

Cahill DG, Ford WK, Goodson KE, et al (2003) Nanoscale thermal transport. J Appl Phys 93:793-818. doi: $10.1063 / 1.1524305$

Carbajal G, Sobhan CB, Peterson GP, et al (2006) Thermal response of a flat heat pipe sandwich structure to a localized heat flux. Int J Heat Mass Transf 49:4070-4081. doi: 10.1016/j.ijheatmasstransfer.2006.03.035

Casimir HBG (1938) Note on the conduction of heat in crystals. Physica 5:495-500. doi: 10.1016/S0031$8914(38) 80162-2$

Chang FL, Hung YM (2017) Dielectric liquid pumping flow in optimally operated micro heat pipes. Int J Heat Mass Transf 108:257-270. doi: 10.1016/j.ijheatmasstransfer.2016.12.018

Chang H-C, Yeo LY (2009) Electrokinetically-Driven Microfluidics and Nanofluidics. Cambridge University Press

Chatterjee A, Derby MM, Peles Y, Jensen MK (2013) Condensation heat transfer on patterned surfaces. Int J Heat Mass Transf 66:889-897. doi: 10.1016/j.ijheatmasstransfer.2013.07.077

Chatterjee A, Derby MM, Peles Y, Jensen MK (2014) Enhancement of condensation heat transfer with patterned surfaces. Int J Heat Mass Transf 71:675-681. doi: 10.1016/j.ijheatmasstransfer.2013.12.069

Chen C-H, Cai Q, Tsai C, et al (2007) Dropwise condensation on superhydrophobic surfaces with two-tier roughness. Appl Phys Lett 90:173108. doi: 10.1063/1.2731434

Chen D, Gelenter MD, Hong M, et al (2017) Icephobic Surfaces Induced by Interfacial Nonfrozen Water. ACS Appl Mater Interfaces 9:4202-4214. doi: 10.1021/acsami.6b13773

Chen G (2005) Nanoscale Energy Transport and Converion. Oxford University Press, boston

Chen R, Lu M-C, Srinivasan V, et al (2009) Nanowires for Enhanced Boiling Heat Transfer. Nano Lett 9:548-553. doi: $10.1021 / \mathrm{n} 18026857$

Chen X, Ye H, Fan X, et al (2016) A review of small heat pipes for electronics. Appl Therm Eng 96:1-17. doi: 
10.1016/j.applthermaleng.2015.11.048

Cheng P, Wu H-Y, Hong F-J (2007) Phase-Change Heat Transfer in Microsystems. J Heat Transfer 129:101. doi: $10.1115 / 1.2410008$

Choi ES, Brooks JS, Eaton DL, et al (2003) Enhancement of thermal and electrical properties of carbon nanotube polymer composites by magnetic field processing. J Appl Phys 94:6034-6039. doi: 10.1063/1.1616638

Collier J. (1972) Covective Boiling and Condensation. McGraw-Hill, New York, New York

Cotter TP (1984a) Principles and prospects for micro heat pipes. In: 5th Intern. Heat Pipe Conf. Tsukuba, Ibaraki, Cotter TP (1984b) Principles and prospects for micro heat pipes. In: 5th Internation Heat Pipe conference. Tsukuba, Ibaraki,

Dai X, Huang X, Yang F, et al (2013) Enhanced nucleate boiling on horizontal hydrophobic-hydrophilic carbon nanotube coatings. Appl Phys Lett 102:161605. doi: 10.1063/1.4802804

Daniel S, Chaudhury MK, Chen JC (2001) Fast Drop Movements Resulting from the Phase Change on a Gradient Surface.

Dhir VK, Abarajith HS, Warrier GR (2005) From Nano to Micro to Macro Scales in Boiling. In: Microscale Heat Transfer Fundamentals and Applications. Springer-Verlag, Berlin/Heidelberg, pp 197-216

DiSalvo FJ (1999) Thermoelectric Cooling and Power Generation.

Dismukes JP, Ekstrom L, Steigmeier EF, et al (1964) Thermal and Electrical Properties of Heavily Doped Ge-Si Alloys up to $1300^{\circ} \mathrm{K}$. J Appl Phys 35:2899-2907. doi: 10.1063/1.1713126

Donadio D, Galli G (2009) Atomistic Simulations of Heat Transport in Silicon Nanowires. Phys Rev Lett 102:195901. doi: 10.1103/PhysRevLett.102.195901

Dresselhaus MS, Chen G, Tang MY, et al (2007) New Directions for Low-Dimensional Thermoelectric Materials. Adv Mater 19:1043-1053. doi: 10.1002/adma.200600527

Eberle P, Tiwari MK, Maitra T, Poulikakos D (2014) Rational nanostructuring of surfaces for extraordinary icephobicity. Nanoscale 6:4874-4881. doi: 10.1039/C3NR06644D

Engemann S, Reichert H, Dosch H, et al (2004) Interfacial Melting of Ice in Contact with SiO 2. Phys Rev Lett 92:205701. doi: 10.1103/PhysRevLett.92.205701

Escher W, Brunschwiler T, Michel B, Poulikakos D (2010a) Experimental Investigation of an Ultrathin Manifold Microchannel Heat Sink for Liquid-Cooled Chips. J Heat Transfer 132:81402. doi: 10.1115/1.4001306 
Escher W, Ghannam R, Khalil A, et al (2010b) Advanced liquid cooling for concentrated photovoltaic electrothermal co-generation. In: 2010 3rd International Conference on Thermal Issues in Emerging Technologies Theory and Applications. IEEE, pp 9-17

Fan X, Zeng G, LaBounty C, et al (2001) SiGeC/Si superlattice microcoolers. Appl Phys Lett 78:1580-1582. doi: $10.1063 / 1.1356455$

Fevre EJL, Rose J. International Heat Transfer Conference 3 - Volume 8, 1966, CONDENSATION - International Heat Transfer Conference Digital Library. In: A THEORY OF HEAT TRANSFER BY DROPWISE CONDENSATION.

Fletcher NH (1958) Size Effect in Heterogeneous Nucleation. J Chem Phys 29:572-576. doi: 10.1063/1.1744540

Forrest E, Williamson E, Buongiorno J, et al (2010) Augmentation of nucleate boiling heat transfer and critical heat flux using nanoparticle thin-film coatings. Int J Heat Mass Transf 53:58-67. doi:

10.1016/j.ijheatmasstransfer.2009.10.008

Gebhart B (2017) Effects of viscous dissipation in natural convection. doi: 10.1017/S0022112062001196

Han C, Sun Q, Li Z, Dou SX (2016) Thermoelectric Enhancement of Different Kinds of Metal Chalcogenides. Adv Energy Mater 6:1600498. doi: 10.1002/aenm.201600498

Hannan MA, Mutashar S, Samad SA, Hussain A (2014) Energy harvesting for the implantable biomedical devices: issues and challenges. 13:1-23. doi: 10.1186/1475-925X-13-79

Harman TC, Spears DL, Walsh MP (1999) PbTe/Te superlattice structures with enhanced thermoelectric figures of merit. J Electron Mater 28:L1-L5. doi: 10.1007/s11664-999-0198-4

Hassan I, Phutthavong P, Abdelgawad M (2004) MICROCHANNEL HEAT SINKS: AN OVERVIEW OF THE STATE-OF-THE-ART. Microscale Thermophys Eng 8:183-205. doi: 10.1080/10893950490477338

Hetsroni G, Mosyak A, Pogrebnyak E, Segal Z (2005) Explosive boiling of water in parallel micro-channels. Int J Multiph Flow 31:371-392. doi: 10.1016/j.ijmultiphaseflow.2005.01.003

Hicks LD, Dresselhaus MS (1993) Effect of quantum-well structures on the thermoelectric figure of merit. Phys Rev B 47:12727-12731. doi: 10.1103/PhysRevB.47.12727

Hicks LD, Harman TC, Dresselhaus MS (1993) Use of quantum-well superlattices to obtain a high figure of merit from nonconventional thermoelectric materials. Appl Phys Lett 63:3230-3232. doi: 10.1063/1.110207 Hochbaum AI, Chen R, Delgado RD, et al (2008) Enhanced thermoelectric performance of rough silicon nanowires. 
Nature 451:163-167. doi: 10.1038/nature06381

Howells MR, Beetz T, Chapman HN, et al (2005) An assessment of the resolution limitation due to radiationdamage in X-ray diffraction microscopy. doi: 10.1016/j.elspec.2008.10.008

Hsu C-C, Chen P-H (2012) Surface wettability effects on critical heat flux of boiling heat transfer using nanoparticle coatings. Int J Heat Mass Transf 55:3713-3719. doi: 10.1016/j.ijheatmasstransfer.2012.03.003

$\mathrm{Hu}$ Y, Shi H, Song H, et al (2013) Effects of a proton scavenger on the thermoelectric performance of free-standing polythiophene and its derivative films. Synth Met 181:23-26. doi: 10.1016/j.synthmet.2013.08.006

Huang Z, Fang H, Zhu J (2007) Fabrication of Silicon Nanowire Arrays with Controlled Diameter, Length, and Density. Adv Mater 19:744-748. doi: 10.1002/adma.200600892

Izumi M, Kumagai S, Shimada R, Yamakawa N (2004) Heat transfer enhancement of dropwise condensation on a vertical surface with round shaped grooves. Exp Therm Fluid Sci 28:243-248. doi: 10.1016/S08941777(03)00046-3

John G. Collier, John R.Thome (1994) CONVECTIVE BOILING AND CONDENSATION, Third Edit. Oxford University Press, Oxford

Jones TB (1973) Electrohydrodynamic heat pipes. Int J Heat Mass Transf 16:1045-1048. doi: 10.1016/00179310(73)90043-4

Jung S, Tiwari MK, Doan NV, Poulikakos D (2012a) Mechanism of supercooled droplet freezing on surfaces. Nat Commun 3:615. doi: 10.1038/ncomms1630

Jung S, Tiwari MK, Poulikakos D (2012b) Frost halos from supercooled water droplets. Proc Natl Acad Sci U S A 109:16073-8. doi: 10.1073/pnas.1206121109

Kandlikar SG (2010) Scale effects on flow boiling heat transfer in microchannels: A fundamental perspective. Int J Therm Sci 49:1073-1085. doi: 10.1016/j.ijthermalsci.2009.12.016

Kandlikar SG, Bapat A V (2017) Evaluation of Jet Impingement, Spray and Microchannel Chip Cooling Options for High Heat Flux Removal. doi: 10.1080/01457630701421703

Kaneko H, Ishiguro T, Takahashi A, Tsukamoto J (1993) Magnetoresistance and thermoelectric power studies of metal-nonmetal transition in iodine-doped polyacetylene. Synth Met 57:4900-4905. doi: 10.1016/03796779(93)90836-L

Kasten P, Zimmermann S, Tiwari MK, et al (2010) HOT WATER COOLED HEAT SINKS FOR EFFICIENT 


\section{DATA CENTER COOLING: TOWARDS ELECTRONIC COOLING WITH HIGH EXERGETIC UTILITY.}

Front Heat Mass Transf. doi: 10.5098/hmt.v1.2.3006

Kemp NT, Kaiser AB, Liu C-J, et al (1999) Thermoelectric power and conductivity of different types of

polypyrrole. J Polym Sci Part B Polym Phys 37:953-960. doi: 10.1002/(SICI)1099-

0488(19990501)37:9<953::AID-POLB7>3.0.CO;2-L

Khonsue O (2012) Experimental on the liquid cooling system with thermoelectric for personal computer. Heat Mass Transf 48:1767-1771. doi: 10.1007/s00231-012-1022-x

Kim D, Kim Y, Choi K, et al (2010a) Improved Thermoelectric Behavior of Nanotube-Filled Polymer Composites with Poly(3,4-ethylenedioxythiophene) Poly(styrenesulfonate). ACS Nano 4:513-523. doi:

$10.1021 / \mathrm{nn} 9013577$

Kim G-H, Shao L, Zhang K, Pipe KP (2013) Engineered doping of organic semiconductors for enhanced thermoelectric efficiency. Nat Mater 12:719-723. doi: 10.1038/nmat3635

Kim H, Kim I, Choi H, Kim W (2010b) Thermal conductivities of Si1-xGex nanowires with different germanium concentrations and diameters. Appl Phys Lett 96:233106. doi: 10.1063/1.3443707

Koo J-M, Im S, Jiang L, Goodson KE (2005) Integrated Microchannel Cooling for Three-Dimensional Electronic Circuit Architectures. J Heat Transfer 127:49. doi: 10.1115/1.1839582

Kraus DA, Abdul A, Welty J Extended Surface Heat Transfer. John Wiley \& Sons, Inc.,

Kreder MJ, Alvarenga J, Kim P, et al (2016) Design of anti-icing surfaces: smooth, textured or slippery? Nat Rev Mater 1:15003. doi: 10.1038/natrevmats.2015.3

Kulinich SA, Farhadi S, Nose K, Du XW (2011) Superhydrophobic Surfaces: Are They Really Ice-Repellent? Langmuir 27:25-29. doi: 10.1021/la104277q

Lan Y, Minnich AJ, Chen G, Ren Z (2010) Enhancement of Thermoelectric Figure-of-Merit by a Bulk Nanostructuring Approach. Adv Funct Mater 20:357-376. doi: 10.1002/adfm.200901512

Le Fevre EJ, Rose JW (1965) An experimental study of heat transfer by dropwise condensation. Int J Heat Mass Transf 8:1117-1133. doi: 10.1016/0017-9310(65)90139-0

Lee EK, Yin L, Lee Y, et al (2012) Large Thermoelectric Figure-of-Merits from SiGe Nanowires by Simultaneously Measuring Electrical and Thermal Transport Properties. Nano Lett 12:2918-2923. doi: 10.1021/nl300587u

Lee J, Mudawar I (2005) Two-phase flow in high-heat-flux micro-channel heat sink for refrigeration cooling 
applications: Part II—heat transfer characteristics. Int J Heat Mass Transf 48:941-955. doi:

10.1016/j.ijheatmasstransfer.2004.09.019

Li C, Peterson GP (2007) Parametric Study of Pool Boiling on Horizontal Highly Conductive Microporous Coated Surfaces. J Heat Transfer 129:1465. doi: 10.1115/1.2759969

Li D, Wu Y, Kim P, et al (2003) Thermal conductivity of individual silicon nanowires. Appl Phys Lett 83:29342936. doi: $10.1063 / 1.1616981$

Lim J, Hippalgaonkar K, Andrews SC, et al (2012) Quantifying Surface Roughness Effects on Phonon Transport in Silicon Nanowires. Nano Lett 12:2475-2482. doi: 10.1021/nl3005868

Lin W, Xiu Y, Jiang H, et al (2008) Self-Assembled Monolayer-Assisted Chemical Transfer of In Situ Functionalized Carbon Nanotubes. J Am Chem Soc 130:9636-9637. doi: 10.1021/ja802142g

Lin W, Zhang R, Moon K-S, Wong CP (2010) Molecular phonon couplers at carbon nanotube/substrate interface to enhance interfacial thermal transport. Carbon N Y 48:107-113. doi: 10.1016/j.carbon.2009.08.033

Lin Y-M, Dresselhaus MS (2003) Thermoelectric properties of superlattice nanowires. Phys Rev B 68:75304. doi: 10.1103/PhysRevB.68.075304

Liu X, Chen Y (2013) Transient thermal performance analysis of micro heat pipes. Appl Therm Eng 58:585-593. doi: 10.1016/j.applthermaleng.2013.04.025

Lu M-C, Chen R, Srinivasan V, et al (2011) Critical heat flux of pool boiling on Si nanowire array-coated surfaces. Int J Heat Mass Transf 54:5359-5367. doi: 10.1016/j.ijheatmasstransfer.2011.08.007

Luckyanova MN, Garg J, Esfarjani K, et al (2012) Coherent Phonon Heat Conduction in Superlattices.

Ma X, Rose JW, Xu D, et al (2000) Advances in dropwise condensation heat transfer: Chinese research. Chem Eng J 78:87-93.

Macner AM, Daniel S, Steen PH (2014) Condensation on Surface Energy Gradient Shifts Drop Size Distribution toward Small Drops. Langmuir 30:1788-1798. doi: 10.1021/la404057g

Maitra T, Antonini C, Tiwari MK, et al (2014) Supercooled Water Drops Impacting Superhydrophobic Textures. Langmuir 30:10855-10861. doi: 10.1021/la502675a

Maitra T, Jung S, Giger ME, et al (2015) Superhydrophobicity vs. Ice Adhesion: The Quandary of Robust Icephobic Surface Design. Adv Mater Interfaces 2:1500330. doi: 10.1002/admi.201500330

Marconnet AM, Panzer MA, Goodson KE (2013) Thermal conduction phenomena in carbon nanotubes and related 
nanostructured materials. Rev Mod Phys 85:1295-1326. doi: 10.1103/RevModPhys.85.1295

Marconnet AM, Yamamoto N, Panzer MA, et al (2011) Thermal Conduction in Aligned Carbon Nanotube-Polymer Nanocomposites with High Packing Density. ACS Nano 5:4818-4825. doi: 10.1021/nn200847u

Mateeva N, Niculescu H, Schlenoff J, Testardi LR (1998) Correlation of Seebeck coefficient and electric conductivity in polyaniline and polypyrrole.

http://oasc12039247realmedia.com/RealMedia/ads/click_lx.ads/www.aip.org/pt/adcenter/pdfcover_test/L37/1952595056/x01/AIP-PT/JAP_ArticleDL_0117/AIP-

2968_JAP_1640x440r2.jpg/434f71374e315a556e61414141774c75?x. doi: 10.1063/1.367119

McGrail BT, Sehirlioglu A, Pentzer E (2015) Polymer Composites for Thermoelectric Applications. Angew Chemie Int Ed 54:1710-1723. doi: 10.1002/anie.201408431

McNamara AJ, Joshi Y, Zhang ZM (2012a) Characterization of nanostructured thermal interface materials - A review. Int J Therm Sci 62:2-11. doi: 10.1016/j.ijthermalsci.2011.10.014

McNamara AJ, Joshi Y, Zhang ZM (2012b) Characterization of nanostructured thermal interface materials - A review. In: International Journal of Thermal Sciences. pp 2-11

Miljkovic N, Enright R, Nam Y, et al (2013a) Jumping-Droplet-Enhanced Condensation on Scalable Superhydrophobic Nanostructured Surfaces. Nano Lett 13:179-187. doi: 10.1021/n1303835d

Miljkovic N, Preston DJ, Enright R, Wang EN (2013b) Electric-Field-Enhanced Condensation on Superhydrophobic Nanostructured Surfaces. ACS Nano 7:11043-11054. doi: 10.1021/nn404707j

Minnich AJ, Dresselhaus MS, Ren ZF, Chen G (2009) Bulk nanostructured thermoelectric materials: current research and future prospects. Energy Environ Sci 2:466. doi: 10.1039/b822664b

Moriarty GP, De S, King PJ, et al (2013) Thermoelectric behavior of organic thin film nanocomposites. J Polym Sci Part B Polym Phys 51:119-123. doi: 10.1002/polb.23186

Murray BJ, O’Sullivan D, Atkinson JD, Webb ME (2012) Ice nucleation by particles immersed in supercooled cloud droplets. Chem Soc Rev 41:6519. doi: 10.1039/c2cs35200a

Narhe RD, Beysens DA (2004) Nucleation and Growth on a Superhydrophobic Grooved Surface. Phys Rev Lett 93:76103. doi: 10.1103/PhysRevLett.93.076103

Nath S, Ahmadi SF, Boreyko JB (2016) A Review of Condensation Frosting. Nanoscale Microscale Thermophys Eng 1-21. doi: 10.1080/15567265.2016.1256007 
Oksman P, Yu S, Kytönen H, Louhenkilpi S (2014) The Effective Thermal Conductivity Method in Continuous Casting of Steel.

Pan Y, Hong G, Raja SN, et al (2015) Significant thermal conductivity reduction of silicon nanowire forests through discrete surface doping of germanium. Appl Phys Lett 106:93102. doi: 10.1063/1.4913879

Pan Y, Tao Y, Qin G, et al (2016) Surface Chemical Tuning of Phonon and Electron Transport in Free-Standing Silicon Nanowire Arrays. Nano Lett 16:6364-6370. doi: 10.1021/acs.nanolett.6b02754

Panigrahi PK (2015) Microscale Convection. In: Transport Phenomena in Microfluidic Systems. John Wiley \& Sons Singapore Pte. Ltd, Singapore, pp 331-374

Paulo J, Gaspar P. (2010) Review and Future Trend of Energy Harvesting Methods for Portable Medical Devices. In: Proceedings of the World Congress on Engineering . London,

Paxson AT, Yagüe JL, Gleason KK, Varanasi KK (2014) Stable Dropwise Condensation for Enhancing Heat Transfer via the Initiated Chemical Vapor Deposition (iCVD) of Grafted Polymer Films. Adv Mater 26:418423. doi: 10.1002/adma.201303065

Pearson JR. Mechanics of Polymer Processing. Elsevier Applied Science Publishers, London and New York,

Peng B, Ma X, Lan Z, et al (2015) Experimental investigation on steam condensation heat transfer enhancement with vertically patterned hydrophobic-hydrophilic hybrid surfaces. Int J Heat Mass Transf 83:27-38. doi: 10.1016/j.ijheatmasstransfer.2014.11.069

Perez-Taborda JA, Rojo MM, Maiz J, et al (2016) Ultra-low thermal conductivities in large-area Si-Ge nanomeshes for thermoelectric applications. doi: 10.1038/srep32778

Pokatilov EP, Nika DL, Balandin AA (2005) Acoustic-phonon propagation in rectangular semiconductor nanowires with elastically dissimilar barriers. Phys Rev B 72:113311. doi: 10.1103/PhysRevB.72.113311

Poudel B, Hao Q, Ma Y, et al (2008) High-Thermoelectric Performance of Nanostructured Bismuth Antimony Telluride Bulk Alloys.

Preston DJ, Mafra DL, Miljkovic N, et al (2015) Scalable Graphene Coatings for Enhanced Condensation Heat Transfer. Nano Lett 15:2902-2909. doi: 10.1021/n1504628s

Qu W, Mudawar I (2003) Flow boiling heat transfer in two-phase micro-channel heat sinks-I. Experimental investigation and assessment of correlation methods. Int J Heat Mass Transf 46:2755-2771. doi: 10.1016/S0017-9310(03)00041-3 
Renfer A, Tiwari MK, Brunschwiler T, et al (2011) Experimental investigation into vortex structure and pressure drop across microcavities in 3D integrated electronics. Exp Fluids 51:731-741. doi: 10.1007/s00348-011$1091-5$

Renfer A, Tiwari MK, Tiwari R, et al (2013) Microvortex-enhanced heat transfer in 3D-integrated liquid cooling of electronic chip stacks. Int J Heat Mass Transf 65:33-43. doi: 10.1016/j.ijheatmasstransfer.2013.05.066

Rice JA, Faghri A (2007) Analysis of Screen Wick Heat Pipes, Including Capillary Dry-Out Limitations. J Thermophys Heat Transf 21:475-486. doi: 10.2514/1.24809

Rose JW (1997) Dropwise condensation theory and experiment: a review. J Eng Thermophys 18:196-200.

Rowe DM, Shukla VS, Savvides N (1981) Phonon scattering at grain boundaries in heavily doped fine-grained silicon-germanium alloys. Nature 290:765-766. doi: 10.1038/290765a0

Schutzius TM, Jung S, Maitra T, et al (2014) Physics of Icing and Rational Design of Surfaces with Extraordinary Icephobicity. Langmuir 31:4807-4821. doi: 10.1021/la502586a

Semenic T, Lin YY, Catton I, Sarraf DB (2008) Use of biporous wicks to remove high heat fluxes. Appl Therm Eng 28:278-283. doi: 10.1016/j.applthermaleng.2006.02.030

Shahil KMF, Balandin AA (2011) Graphene-based thermal interface materials. 2011 11th IEEE Int Conf Nanotechnol 1193-1196. doi: 10.1109/NANO.2011.6144476

Shahil KMF, Balandin AA (2012a) Graphene-multilayer graphene nanocomposites as highly efficient thermal interface materials. Nano Lett 12:861-867. doi: 10.1021/nl203906r

Shahil KMF, Balandin A a. (2012b) Thermal properties of graphene and multilayer graphene: Applications in thermal interface materials. Solid State Commun 152:1331-1340. doi: 10.1016/j.ssc.2012.04.034

Sharma CS, Schlottig G, Brunschwiler T, et al (2015a) A novel method of energy efficient hotspot-targeted embedded liquid cooling for electronics: An experimental study. Int J Heat Mass Transf 88:684-694. doi: 10.1016/j.ijheatmasstransfer.2015.04.047

Sharma CS, Tiwari MK, Michel B, Poulikakos D (2013) Thermofluidics and energetics of a manifold microchannel heat sink for electronics with recovered hot water as working fluid. Int J Heat Mass Transf 58:135-151. doi: 10.1016/j.ijheatmasstransfer.2012.11.012

Sharma CS, Tiwari MK, Zimmermann S, et al (2015b) Energy efficient hotspot-targeted embedded liquid cooling of electronics. Appl Energy 138:414-422. doi: 10.1016/j.apenergy.2014.10.068 
Sootsman JR, Chung DY, Kanatzidis MG (2009) New and Old Concepts in Thermoelectric Materials. Angew Chemie Int Ed 48:8616-8639. doi: 10.1002/anie.200900598

Sun Z, James DK, Tour JM (2011) Graphene Chemistry: Synthesis and Manipulation. J Phys Chem Lett 2:24252432. doi: $10.1021 / \mathrm{jz} 201000 \mathrm{a}$

Takata Y, Hidaka S, Cao JM, et al (2005) Effect of surface wettability on boiling and evaporation. Energy 30:209220. doi: 10.1016/j.energy.2004.05.004

Theofanous TG, Dinh TN, Tu JP, Dinh AT (2002) The boiling crisis phenomenon: Part II: dryout dynamics and burnout. Exp Therm Fluid Sci 26:793-810. doi: 10.1016/S0894-1777(02)00193-0

Tiwari MK, Zimmermann S, Sharma CS, et al (2012) Waste heat recovery in supercomputers and 3D integrated liquid cooled electronics. In: 13th InterSociety Conference on Thermal and Thermomechanical Phenomena in Electronic Systems. IEEE, pp 545-551

Torresin D, Tiwari MK, Del Col D, Poulikakos D (2013) Flow Condensation on Copper-Based Nanotextured Superhydrophobic Surfaces. Langmuir 29:840-848. doi: 10.1021/la304389s

Tourkine P, Le Merrer M, Quéré D (2009) Delayed Freezing on Water Repellent Materials. Langmuir 25:72147216. doi: 10.1021/la900929u

Tuckerman DB, Pease RFW (1981) High-performance heat sinking for VLSI. IEEE Electron Device Lett 2:126129. doi: 10.1109/EDL.1981.25367

Vashaee D, Shakouri A (2007) Thermionic power generation at high temperatures using SiGeSi superlattices. J Appl Phys 101:53719. doi: 10.1063/1.2645607

Venkatasubramanian R, Siivola E, Colpitts T, O’Quinn B (2001) Thin-film thermoelectric devices with high roomtemperature figures of merit. Nature 413:597-602. doi: 10.1038/35098012

Vineis CJ, Shakouri A, Majumdar A, Kanatzidis MG (2010) Nanostructured Thermoelectrics: Big Efficiency Gains from Small Features. Adv Mater 22:3970-3980. doi: 10.1002/adma.201000839

Wang C-C, Chi-Chuan (2017) A Quick Overview of Compact Air-Cooled Heat Sinks Applicable for Electronic Cooling—Recent Progress. Inventions 2:5. doi: 10.3390/inventions2010005

Wang G, Cheng P, Wu H (2007) Unstable and stable flow boiling in parallel microchannels and in a single microchannel. Int J Heat Mass Transf 50:4297-4310. doi: 10.1016/j.ijheatmasstransfer.2007.01.033

Weibel JA, Garimella S V, North MT, et al (2010) Purdue e-Pubs Characterization of Evaporation and Boiling from 
Sintered Powder Wicks Fed by Capillary Action Characterization of evaporation and boiling from sintered powder wicks fed by capillary action. doi: 10.1016/j.ijheatmasstransfer.2010.05.043

Yao Z, Lu Y-W, Kandlikar SG (2011) Effects of nanowire height on pool boiling performance of water on silicon chips. Int J Therm Sci 50:2084-2090. doi: 10.1016/j.ijthermalsci.2011.06.009

Yazicioglu AG, Kakaç S (2010) Convective Heat Transfer in Microscale Slip Flow. Springer Netherlands, pp 15-38

Yoon CO, Reghu M, Moses D, et al (1995) Transports in blends of conducting polymers. Synth Met 69:255-258. doi: 10.1016/0379-6779(94)02439-6

Ziman JM (2001) Electrons and Phonons: The Theory of Transport Phenomena in Solids. Oxford University Press, New York

Zimmermann S, Helmers H, Tiwari MK, et al (2015) A high-efficiency hybrid high-concentration photovoltaic system. Int J Heat Mass Transf 89:514-521. doi: 10.1016/j.ijheatmasstransfer.2015.04.068

Zimmermann S, Meijer I, Tiwari MK, et al (2012a) Aquasar: A hot water cooled data center with direct energy reuse. Energy 43:237-245. doi: 10.1016/j.energy.2012.04.037

Zimmermann S, Tiwari MK, Meijer I, et al (2012b) Hot water cooled electronics: Exergy analysis and waste heat reuse feasibility. Int J Heat Mass Transf 55:6391-6399. doi: 10.1016/j.ijheatmasstransfer.2012.06.027 